\title{
Broken R-parity in the sky and at the LHC
}

\section{S. Bobrovskyi, W. Buchmüller, J. Hajer and J. Schmidt}

Deutsches Elektronen-Synchrotron DESY,

Hamburg, Germany

E-mail: sergei.bobrovskyi@desy.de, buchmuwi@mail.desy.de, jan.hajer@desy.de, jonas.schmidt@desy.de

Abstract: Supersymmetric extensions of the Standard Model with small R-parity and lepton number violating couplings are naturally consistent with primordial nucleosynthesis, thermal leptogenesis and gravitino dark matter. We consider supergravity models with universal boundary conditions at the grand unification scale, and scalar $\tau$-lepton or binolike neutralino as next-to-lightest superparticle (NLSP). Recent Fermi-LAT data on the isotropic diffuse gamma-ray flux yield a lower bound on the gravitino lifetime. Comparing two-body gravitino and neutralino decays we find a lower bound on a neutralino NLSP decay length, $c \tau_{\chi_{1}^{0}} \gtrsim 30 \mathrm{~cm}$. Together with gravitino and neutralino masses one obtains a microscopic determination of the Planck mass. For a $\widetilde{\tau}$-NLSP there exists no modelindependent lower bound on the decay length. Here the strongest bound comes from the requirement that the cosmological baryon asymmetry is not washed out, which yields $c \tau_{\widetilde{\tau}_{1}} \gtrsim 4 \mathrm{~mm}$. However, without fine-tuning of parameters, one finds much larger decay lengths. For typical masses, $m_{3 / 2} \sim 100 \mathrm{GeV}$ and $m_{\mathrm{NLSP}} \sim 150 \mathrm{GeV}$, the discovery of a photon line with an intensity close to the Fermi-LAT limit would imply a decay length $c \tau_{\text {NLSP }}$ of several hundred meters, which can be measured at the LHC.

KEYWORDS: Supersymmetry Phenomenology

ARXIV EPRINT: 1007.5007 


\section{Contents}

$\begin{array}{llr}1 & \text { Introduction } & 1\end{array}$

2 Bilinear R-parity breaking $\quad 2$

3 Spontaneous R-parity breaking 5

4 Neutral, charged and supercurrents $\quad 8$

5 Fermi-LAT and the LHC $\quad 11$

$\begin{array}{lll}5.1 & \text { Neutralino NLSP } & 13\end{array}$

$\begin{array}{lll}5.2 & \widetilde{\tau} \text {-Lepton NLSP } & 15\end{array}$

$\begin{array}{lll}5.3 & \text { Planck mass measurement } & 18\end{array}$

$\begin{array}{lll}6 & \text { Summary and conclusions } & 19\end{array}$

A Gauge and mass eigenstates $\quad 20$

A.1 Mass matrix diagonalization 20

A.2 The currents in mass eigenstate basis 24

\section{Introduction}

Locally supersymmetric extensions of the Standard Model predict the existence of the gravitino, the gauge fermion of supergravity $[1,2]$. For some patterns of supersymmetry breaking, the gravitino is the lightest superparticle (LSP), and therefore a natural dark matter candidate [3]. Heavy unstable gravitinos may cause the 'gravitino problem' [48] for large reheating temperatures in the early universe. This is the case for thermal leptogenesis [9], where gravitino dark matter has become an attractive alternative [10] to the standard WIMP scenario [11].

Recently, it has been shown that models with small R-parity and lepton number breaking naturally yield a consistent cosmology incorporating primordial nucleosynthesis, leptogenesis and gravitino dark matter [12]. The gravitino is no longer stable, but its decays into Standard Model (SM) particles are doubly suppressed by the Planck mass and the small R-parity breaking parameter. Hence, its lifetime can exceed the age of the Universe by many orders of magnitude, and the gravitino remains a viable dark matter candidate [13].

Gravitino decays lead to characteristic signatures in high-energy cosmic rays, in particular to a diffuse gamma-ray flux [12-20]. The recent search of the Fermi-LAT collaboration for monochromatic photon lines [21] and the measurement of the diffuse gamma-ray flux up to photon energies of $100 \mathrm{GeV}$ [22] severely constrain possible signals from decaying 
dark matter. In this paper we study the implications of this data for the decays of the next-to-lightest superparticle (NLSP) at the LHC, extending the estimates in [12].

We shall restrict our analysis to the simplest class of supergravity models with universal boundary conditions at the Grand Unification (GUT) scale, which lead to neutralino or $\widetilde{\tau}$-NLSP. Electroweak precision tests, thermal leptogenesis and gravitino dark matter together allow gravitino and NLSP masses in the range $m_{3 / 2}=10 \ldots 500 \mathrm{GeV}$ and $m_{\mathrm{NLSP}}=100 \ldots 500 \mathrm{GeV}$ [23]. Following [12], the breaking of R-parity is tied to the breaking of lepton number, which leads to a model with bilinear R-parity breaking [24-27]. The soft supersymmetry breaking terms are characteristic for gravity or gaugino mediation.

In order to establish the connection between the gamma-ray flux from gravitino decays and NLSP decays, one needs R-parity breaking matrix elements of neutral, charged and supercurrents. For the considered supergravity models we are able to obtain these matrix elements to good approximation analytically. This makes our results for the NLSP decay lengths rather transparent. As we shall see, the lower bound on the neutralino decay length is a direct consequence of the Fermi-LAT constraints on decaying dark matter. On the other hand, the lower bound on the $\widetilde{\tau}$-decay length is determined by the cosmological bounds on R-parity breaking couplings, which follow from the requirement that the baryon asymmetry is not washed out [29-32].

This paper is organized as follows. In section 2 we discuss the general Lagrangian for R-parity breaking in a basis of scalar SU(2) doublets where all bilinear mixing terms vanish. This leads to new Yukawa and gaugino couplings, some of which are proportional to the up-quark Yukawa couplings. Section 3 deals with the various supersymmetry, R-parity and lepton number breaking terms in the Lagrangian and the relations among them due to a U(1) flavour symmetry of the considered model. The needed R-parity breaking matrix elements of neutral, charged and supercurrent are analytically calculated in section 4, based on the diagonalization of the mass matrices which is discussed in detail in the appendix. The main results of the paper, the bounds on the NLSP decay lengths and the partial decay widths, are described in section 5 , followed by our conclusions in section 6 .

\section{Bilinear R-parity breaking}

Supersymmetric extensions of the Standard Model with bilinear R-parity breaking contain mass mixing terms between lepton and Higgs fields in the superpotential, ${ }^{1}$

$$
\Delta W=\mu_{i} H_{u} l_{i}
$$

as well as the scalar potential induced by supersymmetry breaking,

$$
-\Delta \mathcal{L}=B_{i} H_{u} \tilde{l}_{i}+m_{i d}^{2} \tilde{l}_{i}^{\dagger} H_{d}+\text { h.c. } .
$$

These mixing terms, together with the R-parity conserving superpotential

$$
W=\mu H_{u} H_{d}+h_{i j}^{u} q_{i} u_{j}^{c} H_{u}+h_{i j}^{d} d_{i}^{c} q_{j} H_{d}+h_{i j}^{e} l_{i} e_{j}^{c} H_{d},
$$

\footnotetext{
${ }^{1}$ Our notation for Higgs and matter superfields, scalars and left-handed fermions reads: $H_{u}=\left(H_{u}, h_{u}\right)$, $l_{i}=\left(\tilde{l}_{i}, l_{i}\right)$ etc.
} 
the scalar mass terms

$$
\begin{aligned}
-\mathcal{L}_{\mathrm{M}}= & m_{u}^{2} H_{u}^{\dagger} H_{u}+m_{d}^{2} H_{d}^{\dagger} H_{d}+\left(B H_{u} H_{d}+\text { h.c. }\right) \\
& +\widetilde{m}_{l i}^{2} \tilde{T}_{i}^{\dagger} \tilde{l}_{i}+\widetilde{m}_{e i}^{2} \tilde{e}_{i}^{c \dagger} \tilde{e}_{i}^{c}+\widetilde{m}_{q i}^{2} \tilde{q}_{i}^{\dagger} \tilde{q}_{i}+\widetilde{m}_{u i}^{2} \tilde{u}_{i}^{c \dagger} \tilde{u}_{i}^{c}+\widetilde{m}_{d i}^{2} \tilde{d}_{i}^{c \dagger} \tilde{d}_{i}^{c},
\end{aligned}
$$

and the standard $\mathrm{SU}(3) \times \mathrm{SU}(2) \times \mathrm{U}(1)_{Y}$ gauge interactions define the supersymmetric standard model with bilinear R-parity breaking. Note that the Higgs mass terms $m_{u}^{2}$ and $m_{d}^{2}$ contain the contributions from the superpotential (2.3) and the soft supersymmetry breaking terms. For simplicity, we have assumed flavour diagonal mass matrices in (2.4).

For a generic choice of parameters the electroweak symmetry is broken by vacuum expectation values (VEVs) of all scalar $\mathrm{SU}(2)$ doublets,

$$
\left\langle H_{u}^{0}\right\rangle=v_{u}, \quad\left\langle H_{d}^{0}\right\rangle=v_{d}, \quad\left\langle\tilde{\nu}_{i}\right\rangle=v_{i}
$$

with $^{2}$

$$
\frac{v_{u}}{v_{d}} \equiv \tan \beta, \quad \widehat{\epsilon}_{i} \equiv \frac{v_{i}}{v_{d}}=\frac{B_{i} \tan \beta-m_{i d}^{2}-\mu \mu_{i}^{*}}{\widetilde{m}_{l i}^{2}+\frac{1}{2} m_{Z}^{2} \cos 2 \beta},
$$

where higher order terms in the R-parity breaking parameters have been neglected.

It is convenient to discuss the predictions of the model in a basis of $\mathrm{SU}(2)$ doublets where the mass mixings $\mu_{i}, B_{i}$ and $m_{i d}^{2}$ in eqs. (2.1) and (2.2) are traded for R-parity breaking Yukawa coulings. This can easily be achieved by field redefinitions. First one rotates the superfields $H_{d}$ and $l_{i}$,

$$
H_{d}=H_{d}^{\prime}-\epsilon_{i} l_{i}^{\prime}, \quad l_{i}=l_{i}^{\prime}+\epsilon_{i} H_{d}^{\prime}, \quad \epsilon_{i}=\frac{\mu_{i}}{\mu} .
$$

Then the bilinear term (2.1) vanishes for the new fields, i.e., $\mu_{i}^{\prime}=0$, and one obtains instead the cubic R-parity violating terms

$$
\Delta W^{\prime}=\frac{1}{2} \lambda_{i j k} l_{i}^{\prime} e_{j}^{c} l_{k}^{\prime}+\lambda_{i j k}^{\prime} d_{i}^{c} q_{j} l_{k}^{\prime},
$$

where

$$
\lambda_{i j k}=-h_{i j}^{e} \epsilon_{k}+h_{k j}^{e} \epsilon_{i}, \quad \lambda_{i j k}^{\prime}=-h_{i j}^{d} \epsilon_{k} .
$$

The new R-parity breaking mass mixings are given by

$$
B_{i}^{\prime}=B_{i}-B \epsilon_{i}, \quad m_{i d}^{2 \prime}=m_{i d}^{2}+\epsilon_{i}\left(\widetilde{m}_{l i}^{2}-m_{d}^{2}\right) .
$$

The corrections for R-parity conserving mass terms are negligable.

In a second step one can perform a non-supersymmetric rotation among all scalar SU(2) doublets,

$$
H_{d}^{\prime}=H_{d}^{\prime \prime}-\epsilon_{i}^{\prime} \tilde{l}_{i}^{\prime \prime}, \quad \varepsilon H_{u}^{*}=\varepsilon H_{u}^{*}-\epsilon_{i}^{\prime \prime} \tilde{l}_{i}^{\prime \prime}, \quad \tilde{l}_{i}^{\prime}=\tilde{l}_{i}^{\prime \prime}+\epsilon_{i}^{\prime} H_{d}^{\prime \prime}+\epsilon_{i}^{\prime \prime} \varepsilon H_{u}^{*},
$$

\footnotetext{
${ }^{2}$ Note that our result for $\widehat{\epsilon}_{i}=v_{i} / v_{d}$ holds at all renormalization scales, contrary to different expressions used in the literature.
} 
where $\varepsilon$ is the usual $\mathrm{SU}(2)$ matrix, $\varepsilon=i \tau^{2}$. Choosing

$$
\begin{aligned}
\epsilon_{i}^{\prime} & =-\frac{B_{i}^{\prime} B+m_{i d}^{2 \prime}\left(\widetilde{m}_{l i}^{2}-m_{u}^{2}\right)}{\left(\widetilde{m}_{l i}^{2}-m_{u}^{2}\right)\left(\widetilde{m}_{l i}^{2}-m_{d}^{2}\right)-B^{2}}, \\
\epsilon_{i}^{\prime \prime} & =\frac{B_{i}^{\prime}\left(\widetilde{m}_{l i}^{2}-m_{d}^{2}\right)+B m_{i d}^{2 \prime}}{\left(\widetilde{m}_{l i}^{2}-m_{u}^{2}\right)\left(\widetilde{m}_{l i}^{2}-m_{d}^{2}\right)-B^{2}},
\end{aligned}
$$

the $H_{u} \tilde{l}_{i}$ and $\tilde{l}^{\dagger} H_{d}$ mixing terms vanish in the new basis of doublets. According to (2.6) also the scalar lepton VEVs $\left\langle\tilde{\nu}_{i}\right\rangle$ vanish in this basis.

It is straightforward to work out the R-parity violating Yukawa couplings which are induced by the rotation (2.11). We are particularly interested in the terms containing one light superparticle, i.e, a scalar lepton, bino or wino. The corresponding couplings read, after dropping prime and double-prime superscripts on all fields, ${ }^{3}$

$$
\begin{aligned}
-\Delta \mathcal{L} \supset & \frac{1}{2} \lambda_{i j k} l_{i} \tilde{e}_{j}^{c} l_{k}+\lambda_{i j k}^{\prime} d_{i}^{c} q_{j} \tilde{l}_{k}+\hat{\lambda}_{i j k} l_{i} e_{j}^{c} \tilde{l}_{k}+\hat{\lambda}_{i j k}^{\prime} q_{i} u_{j}^{c} \varepsilon \tilde{l}_{k}^{*} \\
& +h_{i j}^{e}\left(\epsilon_{i}^{\prime} H_{d}+\epsilon_{i}^{\prime \prime} \varepsilon H_{u}^{*}\right) e_{j}^{c} h_{d} \\
& -\frac{g^{\prime}}{\sqrt{2}}\left(\epsilon_{i}^{\prime} H_{d}^{\dagger}-\epsilon_{i}^{\prime \prime} H_{u}^{T} \varepsilon\right) l_{i} b+\frac{g}{\sqrt{2}}\left(\epsilon_{i}^{\prime} H_{d}^{\dagger}-\epsilon_{i}^{\prime \prime} H_{u}^{T} \varepsilon\right) \tau^{I} l_{i} w^{I}+\text { h.c. },
\end{aligned}
$$

where the Yukawa couplings are given by

$$
\begin{array}{ll}
\lambda_{i j k}=-h_{i j}^{e} \epsilon_{k}+h_{k j}^{e} \epsilon_{i}, & \lambda_{i j k}^{\prime}=-h_{i j}^{d}\left(\epsilon_{k}+\epsilon_{k}^{\prime}\right), \\
\hat{\lambda}_{i j k}=-h_{i j}^{e}\left(\epsilon_{k}+\epsilon_{k}^{\prime}\right)+h_{k j}^{e} \epsilon_{i}, & \hat{\lambda}_{i j k}^{\prime}=h_{i j}^{u} \epsilon_{k}^{\prime \prime} .
\end{array}
$$

Since the field transformations are non-supersymmetric, the couplings $\lambda_{i j k}$ and $\hat{\lambda}_{i j k}$ are no longer equal as in eq. (2.9). Furthermore, a new coupling of right-handed up-quarks, $\hat{\lambda}_{i j k}^{\prime}$, has been generated.

After electroweak symmetry breaking one obtains new mass mixings between higgsinos, gauginos and leptons,

$$
-\Delta \mathcal{L}_{M} \supset m_{i j}^{e} \frac{\zeta_{i}}{c_{\beta}} e_{j}^{c} h_{d}-m_{Z} s_{w} \zeta_{i}^{*} \nu_{i} b+m_{Z} c_{w} \zeta_{i}^{*} \nu_{i} w^{3}+\text { h.c. },
$$

where we have defined

$$
\begin{aligned}
\zeta_{i} & =\frac{\epsilon_{i}^{\prime} v_{d}+\epsilon_{i}^{\prime \prime} v_{u}}{v}, & v & =\sqrt{v_{u}^{2}+v_{d}^{2}}, \\
m_{i j}^{e} & =h_{i j}^{e} v_{d}, & \frac{v_{u}}{v_{d}} & =\tan \beta \equiv \frac{s_{\beta}}{c_{\beta}}, \\
m_{Z} & =\frac{\sqrt{g^{2}+g^{\prime 2}} v}{\sqrt{2}}, & s_{w} & =\frac{g^{\prime}}{\sqrt{g^{2}+g^{\prime 2}}}=\sqrt{1-c_{w}^{2}} .
\end{aligned}
$$

Given the Yukawa couplings $h_{i j}^{u}, h_{i j}^{d}$ and $h_{i j}^{e}$, the Lagrangian (2.14) predicts $108 \mathrm{R}$ parity breaking Yukawa couplings in terms of 9 independent parameters which may be chosen as

$$
\mu_{i}, B_{i}, m_{i d}^{2} \quad \text { or } \epsilon_{i}, \epsilon_{i}^{\prime}, \epsilon_{i}^{\prime \prime} .
$$

\footnotetext{
${ }^{3}$ Our notation for gauge fields, field strengths and left-handed gauginos reads: $B_{\mu}, B_{\mu \nu}, b$ etc.
} 


\begin{tabular}{|l|cccccccc|}
\hline & $\Psi$ & $H_{u}$ & $H_{d}$ & $N$ & $N^{c}$ & $\Phi$ & $X$ & $Z$ \\
\hline$R$ & 1 & 0 & 0 & 0 & -2 & -1 & 4 & 0 \\
\hline
\end{tabular}

Table 1. R-charges of matter fields $\Psi=q, u^{c}, e^{c}, d^{c}, l, \nu^{c}$, Higgs fields and gauge singlets.

These parameters determine lepton-gaugino mass mixings, lepton-slepton and quarkslepton Yukawa couplings, and therefore the low-energy phenomenology. The values of these parameters depend on the pattern of supersymmetry breaking and the flavour structure of the supersymmetric standard model.

\section{Spontaneous R-parity breaking}

Let us now compute the parameters $\epsilon_{i}, \epsilon_{i}^{\prime}$ and $\epsilon_{i}^{\prime \prime}$ in a specific example where the spontaneous breaking of R-parity is related to the spontaneous breaking of B-L, the difference of baryon and lepton number [12].

We consider a supersymmetric extension of the standard model with symmetry group

$$
G=\mathrm{SU}(3) \times \mathrm{SU}(2) \times \mathrm{U}(1)_{Y} \times \mathrm{U}(1)_{B-L} \times \mathrm{U}(1)_{R} .
$$

In addition to three quark lepton generations and the Higgs fields $H_{u}$ and $H_{d}$ the model contains three right-handed neutrinos $\nu_{i}^{c}$, two non-Abelian singlets $N^{c}$ and $N$, which transform as $\nu^{c}$ and its complex conjugate, respectively, and three gauge singlets $X, \Phi$ and $Z$. The part of the superpotential responsible for neutrino masses has the usual form

$$
W_{\nu}=h_{i j}^{\nu} l_{i} \nu_{j}^{c} H_{u}+\frac{1}{M_{\mathrm{P}}} h_{i j}^{n} \nu_{i}^{c} \nu_{j}^{c} N^{2}
$$

where $M_{\mathrm{P}}=2.4 \times 10^{18} \mathrm{GeV}$ is the Planck mass. The expectation value of $H_{u}$ generates Dirac neutrino masses, whereas the expectation value of the singlet Higgs field $N$ generates the Majorana mass matrix of the right-handed neutrinos $\nu_{i}^{c}$. The superpotential responsible for B-L breaking is chosen as

$$
W_{B-L}=X\left(N N^{c}-\Phi^{2}\right),
$$

where unknown Yukawa couplings have been set equal to one. $\Phi$ plays the role of a spectator field, which will finally be replaced by its expectation value, $\langle\Phi\rangle=v_{B-L}$. Similarly, $Z$ is a spectator field which breaks supersymmetry and $\mathrm{U}(1)_{R},\langle Z\rangle=F_{Z} \theta \theta$. The superpotential in eqs. (3.2) and (3.3) is the most general one consistent with the R-charges listed in table 1 , up to nonrenormalizable terms which are irrelevant for our discussion.

The expectation value of $\Phi$ leads to the breaking of $B-L$,

$$
\langle N\rangle=\left\langle N^{c}\right\rangle=\langle\Phi\rangle=v_{B-L},
$$

where the first equality is a consequence of the $\mathrm{U}(1)_{B-L} \mathrm{D}$-term. This generates a Majorana mass matrix $M$ for the right-handed neutrinos with three large eigenvalues $M_{3}>M_{2}>M_{1}$. If the largest eigenvalue of $h^{n}$ is $\mathcal{O}(1)$, one has $M_{3} \simeq v_{B-L}^{2} / M_{\mathrm{P}}$. Integrating out the heavy 
Majorana neutrinos one obtains the familiar dimension-5 seesaw operator which yields the light neutrino masses.

Since the field $\Phi$ carries R-charge -1 , the VEV $\langle\Phi\rangle$ breaks R-parity, which is conserved by the VEV $\langle Z\rangle$. Thus, the breaking of $B-L$ is tied to the breaking of R-parity, which is then transmitted to the low-energy degrees of freedom via higher-dimensional operators in the superpotential and the Kähler potential. Bilinear R-parity breaking, as discussed in the previous section, is obtained from a correction to the Kähler potential,

$$
\begin{aligned}
\Delta K= & \frac{1}{M_{\mathrm{P}}{ }^{3}}\left(a_{i} Z^{\dagger} \Phi^{\dagger} N^{c} H_{u} l_{i}+a_{i}^{\prime} Z^{\dagger} \Phi N^{\dagger} H_{u} l_{i}\right) \\
+\frac{1}{M_{\mathrm{P}}{ }^{4}} & \left(b_{i} Z^{\dagger} Z \Phi^{\dagger} N^{c} H_{u} l_{i}+b_{i}^{\prime} Z^{\dagger} Z \Phi N^{\dagger} H_{u} l_{i}\right. \\
& \left.\quad+c_{i} Z^{\dagger} Z \Phi^{\dagger} N^{c} l_{i}^{\dagger} H_{d}+c_{i}^{\prime} Z^{\dagger} Z \Phi N^{\dagger} l_{i}^{\dagger} H_{d}\right)+ \text { h.c. }
\end{aligned}
$$

Replacing the spectator fields $Z$ and $\Phi$, as well as $N^{c}$ and $N$ by their expectation values, one obtains the correction to the superpotential

$$
\Delta W=\mu_{i} H_{u} l_{i},
$$

with

$$
\mu_{i}=\sqrt{3}\left(a_{i}+a_{i}^{\prime}\right) m_{3 / 2} \Theta, \quad \Theta=\frac{v_{B-L}^{2}}{M_{\mathrm{P}}^{2}} \simeq \frac{M_{3}}{M_{\mathrm{P}}},
$$

where $m_{3 / 2}=F_{Z} /\left(\sqrt{3} M_{\mathrm{P}}\right)$ is the gravitino mass. Note that $\Theta$ can be increased or decreased by including appropriate Yukawa couplings in eqs. (3.2) and (3.3). The corresponding corrections to the scalar potential are given by

$$
-\Delta \mathcal{L}=B_{i} H_{u} \tilde{l}_{i}+m_{i d}^{2} \tilde{l}_{i}^{\dagger} H_{d}+\text { h.c. },
$$

where

$$
B_{i}=3\left(b_{i}+b_{i}^{\prime}\right) m_{3 / 2}^{2} \Theta, \quad m_{i d}^{2}=3\left(c_{i}+c_{i}^{\prime}\right) m_{3 / 2}^{2} \Theta .
$$

The corresponding R-parity conserving terms are generated by [33]

$$
K \supset \frac{a_{0}}{M_{\mathrm{P}}} Z^{\dagger} H_{u} H_{d}+\frac{b_{0}}{M_{\mathrm{P}}^{2}} Z^{\dagger} Z H_{u} H_{d}+\text { h.c. },
$$

which yields

$$
\begin{array}{rlrl}
W & \supset \mu H_{u} H_{d}, & & \mu \sqrt{3} a_{0} m_{3 / 2}, \\
-\mathcal{L} \supset B H_{u} H_{d}+\text { h.c. }, & & B=3 b_{0} m_{3 / 2}^{2} .
\end{array}
$$

Higher dimensional operators yield further R-parity violating couplings between scalars and fermions. However, the cubic couplings allowed by the symmetries of our model are suppressed by one power of $M_{\mathrm{P}}$ compared to ordinary Yukawa couplings and cubic soft supersymmetry breaking terms. Note that the coefficients of the nonrenormalizable operators are free parameters, which are only fixed in specific models of supersymmetry breaking. In particular, one may have $\mu^{2}, \widetilde{m}_{i}^{2}>m_{3 / 2}^{2}$ and hence a gravitino LSP. All parameters 


\begin{tabular}{|c|cccccccccccccc|}
\hline$\psi_{i}$ & $\mathbf{1 0}_{3}$ & $\mathbf{1 0}_{2}$ & $\mathbf{1 0}_{1}$ & $\mathbf{5}_{3}^{*}$ & $\mathbf{5}_{2}^{*}$ & $\mathbf{5}_{1}^{*}$ & $\nu_{3}^{c}$ & $\nu_{2}^{c}$ & $\nu_{1}^{c}$ & $H_{u}$ & $H_{d}$ & $\Phi$ & $X$ & $Z$ \\
\hline$Q_{i}$ & 0 & 1 & 2 & 1 & 1 & 2 & 0 & 0 & 1 & 0 & 0 & 0 & 0 & 0 \\
\hline
\end{tabular}

Table 2. Chiral U(1) charges. $\mathbf{1 0}_{i}=\left(q_{i}, u_{i}^{c}, e_{i}^{c}\right), \mathbf{5}=\left(d_{i}^{c}, l_{i}\right), i=1 \ldots 3$.

are defined at the GUT scale and have to be evolved to the electroweak scale by the renormalization group equations.

The phenomenological viability of the model depends on the size of R-parity breaking mass mixings and therefore on the scale $v_{B-L}$ of R-parity breaking as well as the parameters $a_{i} \ldots c_{i}^{\prime}$ in eq. (3.5). Any model of flavour physics, which predicts Yukawa couplings, will generically also predict the parameters $a_{i} \ldots c_{i}^{\prime}$. As a typical example, we use a model [34] for quark and lepton mass hierarchies based on a Froggatt-Nielsen U(1) flavour symmetry, which is consistent with thermal leptogenesis and all contraints from flavour changing processes [35].

The mass hierarchy is generated by the expectation value of a singlet field $\phi$ with charge $Q_{\phi}=-1$ via nonrenormalizable interactions with a scale $\Lambda=\langle\phi\rangle / \eta>\Lambda_{G U T}, \eta \simeq 0.06$. The $\eta$-dependence of Yukawa couplings and bilinear mixing terms for multiplets $\psi_{i}$ with charges $Q_{i}$ is given by

$$
h_{i j} \propto \eta^{Q_{i}+Q_{j}}, \quad \mu_{i} \propto \eta^{Q_{i}}, \quad B_{i} \propto \eta^{Q_{i}}, \quad m_{i d}^{2} \propto \eta^{Q_{i}} .
$$

The charges $Q_{i}$ for quarks, leptons, Higgs fields and singlets are listed in table 2. The neutrino mass scale $m_{\nu} \simeq 0.01 \mathrm{eV}$ implies for the heaviest right-handed neutrinos $M_{2} \sim$ $M_{3} \sim 10^{12} \mathrm{GeV}$. The corresponding scales for $B-L$ breaking and R-parity breaking are

$$
v_{B-L} \simeq 10^{15} \mathrm{GeV}, \quad \Theta=\frac{v_{B-L}^{2}}{M_{\mathrm{P}}^{2}} \simeq 10^{-6} .
$$

For the small R-parity breaking considered in this paper the neutrino masses are dominated by the conventional seesaw contribution [12].

The R-parity breaking parameters $\mu_{i}, B_{i}$ and $m_{i d}^{2}$ strongly depend on the mechanism of supersymmetry breaking. In the example considered in this section all mass parameters are $\mathcal{O}\left(m_{3 / 2}\right)$, which corresponds to gravity or gaugino mediation. From eqs. (3.6), (3.7) and (3.11) one reads off

$$
\mu_{i}=\hat{a} \eta^{Q_{i}} m_{3 / 2} \Theta, \quad B_{i}=\hat{b} \eta^{Q_{i}} m_{3 / 2}^{2} \Theta, \quad m_{i d}^{2}=\hat{c} \eta^{Q_{i}} m_{3 / 2}^{2} \Theta
$$

with $\hat{a}, \hat{b}, \hat{c}=\mathcal{O}(1)$. Correspondingly, one obtains for $\epsilon$-parameters (cf. (2.12), (2.13))

$$
\epsilon_{i}=a \eta^{Q_{i}} \Theta, \quad \epsilon_{i}^{\prime}=b \eta^{Q_{i}} \Theta, \quad \epsilon_{i d}^{\prime \prime}=c \eta^{Q_{i}} \Theta
$$

with $a, b, c=\mathcal{O}(1)$. Our phenomenological analysis in section 5.2 will be based on this parametrization of bilinear R-parity breaking.

Depending on the mechanism of supersymmetry breaking, the R-parity breaking soft terms may vanish at the GUT scale [25-27],

$$
B_{i}\left(\Lambda_{\mathrm{GUT}}\right)=m_{i d}^{2}\left(\Lambda_{\mathrm{GUT}}\right)=0 .
$$


Non-zero values of these parameters at the electroweak scale are then induced by radiative corrections. The renormalization group equations for the bilinear R-parity breaking mass terms read (cf. [25-27], $t=\ln \Lambda$ ):

$$
\begin{aligned}
16 \pi^{2} \frac{d \mu_{i}}{d t}= & 3 \mu_{i}\left(h_{j k}^{u} h_{j k}^{u *}-\frac{1}{5} g_{1}^{2}-g_{2}^{2}\right)+\mu_{k} h_{i j}^{e} h_{k j}^{e *}-\mu\left(\lambda_{i j k} h_{k j}^{e *}+3 \lambda_{k j i}^{\prime} h_{k j}^{d *}\right), \\
16 \pi^{2} \frac{d B_{i}}{d t}= & 3 B_{i}\left(h_{j k}^{u} h_{j k}^{u *}-\frac{1}{5} g_{1}^{2}-g_{2}^{2}\right)+6 \mu_{i}\left(\frac{1}{5} g_{1}^{2} M_{1}+g_{2}^{2} M_{2}\right) \\
& +B_{k} h_{i j}^{e} h_{k j}^{e *}-B\left(\lambda_{i j k} h_{k j}^{e *}+3 \lambda_{k j i}^{\prime} h_{k j}^{d *}\right) \\
16 \pi^{2} \frac{d m_{i d}^{2}}{d t}= & \lambda_{k j i}^{*} h_{k j}^{e} m_{d}^{2}-m_{j d}^{2} h_{j k}^{e} h_{i k}^{e *}-3 \lambda_{k j i}^{\prime} h_{k j}^{d} m_{d}^{2}+h_{j k}^{e} h_{j k}^{e *} m_{i d}^{2} \\
& +3 h_{k j}^{d *} h_{k j}^{d} m_{i d}^{2}+\widetilde{m}_{l i}^{2} \lambda_{n k i}^{*} h_{n k}^{e}-3 \widetilde{m}_{l i}^{2} \lambda_{n k i}^{*} h_{n k}^{e} \\
& +2 \lambda_{k j i}^{*} \widetilde{m}_{l k}^{2} \lambda_{k j}+2 \lambda_{k j i}^{*} h_{k j}^{e} \widetilde{m}_{e j}^{2}-6 \lambda_{k j i}^{\prime *} h_{k j}^{d} \widetilde{m}_{d k}^{2}-6 \lambda_{k j i}^{\prime *} \widetilde{m}_{q j}^{2} h_{k j}^{d} .
\end{aligned}
$$

In bilinear R-parity breaking, the R-parity violating Yukawa couplings vanish at the GUT scale. One-loop radiative corrections then yield for the soft terms at the electroweak scale (cf. eqs. (3.16), (3.17); $\left.\epsilon_{i}=\mu_{i} / \mu\right)$ :

$$
B_{i}\left(\Lambda_{\mathrm{EW}}\right)=\frac{\mu_{i}}{16 \pi^{2}}\left(\frac{6}{5} g^{\prime 2} M_{1}+6 g^{2} M_{2}\right) \ln \frac{\Lambda_{\mathrm{GUT}}}{\Lambda_{\mathrm{EW}}}, \quad m_{i d}^{2}\left(\Lambda_{\mathrm{EW}}\right)=0 .
$$

This illustrates that the bilinear R-parity breaking terms $\mu_{i}^{2}, B_{i}$ and $m_{i d}^{2}$ are not necessarily of the same order of magnitude at the electroweak scale.

\section{Neutral, charged and supercurrents}

In section 2 we have discussed the R-parity breaking Yukawa couplings in our model. For a phenomenological analysis we also need the couplings of the gauge fields, i.e., photon, W-bosons and gravitino, to charged and neutral matter,

$$
\mathcal{L}=-e J_{e \mu} A^{\mu}-\frac{g}{c_{w}} J_{Z \mu} Z^{\mu}-\frac{g}{\sqrt{2}} J_{\mu}^{-} W^{+\mu}-\frac{g}{\sqrt{2}} J_{\mu}^{+} W^{-\mu}-\frac{1}{2 M_{\mathrm{P}}} \bar{\psi}_{\mu} S^{\mu} .
$$

The corresponding currents read

$$
\begin{aligned}
J_{e \mu}= & \bar{w}^{+} \gamma_{\mu} w^{+}-\bar{w}^{-} \gamma_{\mu} w^{-}-\bar{e}_{i} \gamma_{\mu} e_{i}+\bar{e}_{i}^{c} \gamma_{\mu} e_{i}^{c}-\bar{h}_{d}^{-} \gamma_{\mu} h_{d}^{-}+\bar{h}_{u}^{+} \gamma_{\mu} h_{u}^{+}, \\
J_{Z \mu}= & \bar{w}^{+} \gamma_{\mu} w^{+}-\bar{w}^{-} \gamma_{\mu} w^{-}+\frac{1}{2} \bar{\nu}_{i} \gamma_{\mu} \nu_{i}-\frac{1}{2} \bar{e}_{i} \gamma_{\mu} e_{i} \\
& +\frac{1}{2} \bar{h}_{d}^{0} \gamma_{\mu} h_{d}^{0}-\frac{1}{2} \bar{h}_{d}^{-} \gamma_{\mu} h_{d}^{-}+\frac{1}{2} \bar{h}_{u}^{+} \gamma_{\mu} h_{u}^{+}-\frac{1}{2} \bar{h}_{u}^{0} \gamma_{\mu} h_{u}^{0}-s_{w}^{2} J_{e \mu}, \\
J_{\mu}^{-}= & \sqrt{2}\left(\bar{w}^{3} \gamma_{\mu} w^{-}-\bar{w}^{+} \gamma_{\mu} w^{3}\right)+\bar{\nu}_{i} \gamma_{\mu} e_{i}+\bar{h}_{d}^{0} \gamma_{\mu} h_{d}^{-}+\bar{h}_{u}^{+} \gamma_{\mu} h_{u}^{0}, \\
S^{\mu}= & \frac{i}{4}\left[\gamma^{\nu}, \gamma^{\rho}\right] \gamma^{\mu}\left(\widetilde{b} B_{\nu \rho}+\widetilde{w}^{I} W_{\nu \rho}^{I}\right)+\ldots
\end{aligned}
$$

The gravitino and the gauginos are now Majorana fermions,

$$
\widetilde{b}=b+b^{c}, \quad \widetilde{w}^{I}=w^{I}+w^{c I},
$$


where the superscript $c$ denotes charge conjugation. In eqs. (4.2)-(4.5) we have only listed contributions to the currents which will be relevant in our phenomenological analysis.

The R-parity breaking described in the previous section leads to mass mixings between the neutralinos $b, w^{3}, h_{u}^{0}, h_{d}^{0}$ with the neutrinos $\nu_{i}$, and the charginos $w^{+}, h_{u}^{+}, w^{-}, h_{d}^{-}$with the charged leptons $e_{i}^{c}, e_{i}$, respectively. The $7 \times 7$ neutralino mass matrix reads in the gauge eigenbasis

$$
\mathcal{M}^{N}=\left(\begin{array}{ccccc}
M_{1} & 0 & m_{Z} s_{\beta} s_{w} & -m_{Z} c_{\beta} s_{w} & -\zeta_{i} m_{Z} s_{w} \\
0 & M_{2} & -m_{Z} s_{\beta} c_{w} & m_{Z} c_{\beta} c_{w} & \zeta_{i} m_{Z} c_{w} \\
m_{Z} s_{\beta} s_{w} & -m_{Z} s_{\beta} c_{w} & 0 & -\mu & 0 \\
-m_{Z} c_{\beta} s_{w} & m_{Z} c_{\beta} c_{w} & -\mu & 0 & 0 \\
-\zeta_{i} m_{Z} s_{w} & \zeta_{i} m_{Z} c_{w} & 0 & 0 & 0
\end{array}\right)
$$

where we have neglected neutrino masses. Correspondingly, the $5 \times 5$ chargino mass matrix which connects the states $\left(w^{-}, h_{d}^{-}, e_{i}\right)$ and $\left(w^{+}, h_{u}^{+}, e_{i}^{c}\right)$ is given by

$$
\mathcal{M}^{C}=\left(\begin{array}{ccccc}
M_{2} & m_{Z} s_{\beta} c_{w} & 0 & 0 & 0 \\
m_{Z} c_{\beta} c_{w} & \mu & \zeta_{1} h_{11}^{e} \mu & \zeta_{2} h_{22}^{e} \mu & \zeta_{3} h_{33}^{e} \mu \\
\zeta_{1} m_{Z} c_{w} & 0 & h_{11}^{e} v c_{\beta} & 0 & 0 \\
\zeta_{2} m_{Z} c_{w} & 0 & 0 & h_{22}^{e} v c_{\beta} & 0 \\
\zeta_{3} m_{Z} c_{w} & 0 & 0 & 0 & h_{33}^{e} v c_{\beta}
\end{array}\right)
$$

Note that all gaugino and higgsino mixings with neutrinos and charged leptons are parametrized by the three parameters $\zeta_{i}$.

In the following section we shall need the couplings of gravitino, $W$ - and $Z$-bosons to neutralino and chargino mass eigenstates. Since $\zeta_{i} \ll 1$, diagonalization of the mass matrices to first order in $\zeta_{i}$ is obviously sufficient. We shall also consider supergravity models where the supersymmetry breaking parameters satisfy the inequalities (cf. figure 1)

$$
m_{Z}<M_{1,2}<\mu \text {. }
$$

The gaugino-higgsino mixings are $\mathcal{O}\left(m_{Z} / \mu\right)$, and therefore suppressed, and $\chi_{1}^{0}$, the lightest neutralino, is bino-like.

The mass matrices $\mathcal{M}^{N}$ and $\mathcal{M}^{C}$ are diagonalized by unitary and bi-unitary transformations, respectively,

$$
U^{(n) T} \mathcal{M}^{N} U^{(n)}=\mathcal{M}_{\text {diag }}^{N}, \quad U^{(c) \dagger} \mathcal{M}^{C} \widetilde{U}^{(c)}=\mathcal{M}_{\text {diag }}^{C}
$$

where $U^{(n) \dagger} U^{(n)}=U^{(c) \dagger} U^{(c)}=\widetilde{U}^{(c) \dagger} \widetilde{U}^{(c)}=1$. These unitary transformations relate the neutral and charged gauge eigenstates to the mass eigenstates $\left(\chi_{a}^{0}, \nu_{i}^{\prime}\right)(a=1, \ldots, 4)$ and $\left(\chi_{\alpha}^{-}, e_{i}^{\prime}\right),\left(\chi_{\alpha}^{+}, e_{i}^{c^{\prime}}\right)(\alpha=1,2)$, respectively. Inserting these transformations in eqs. (4.3)(4.5) and dropping prime superscripts, one obtains neutral, charged and supercurrents in 


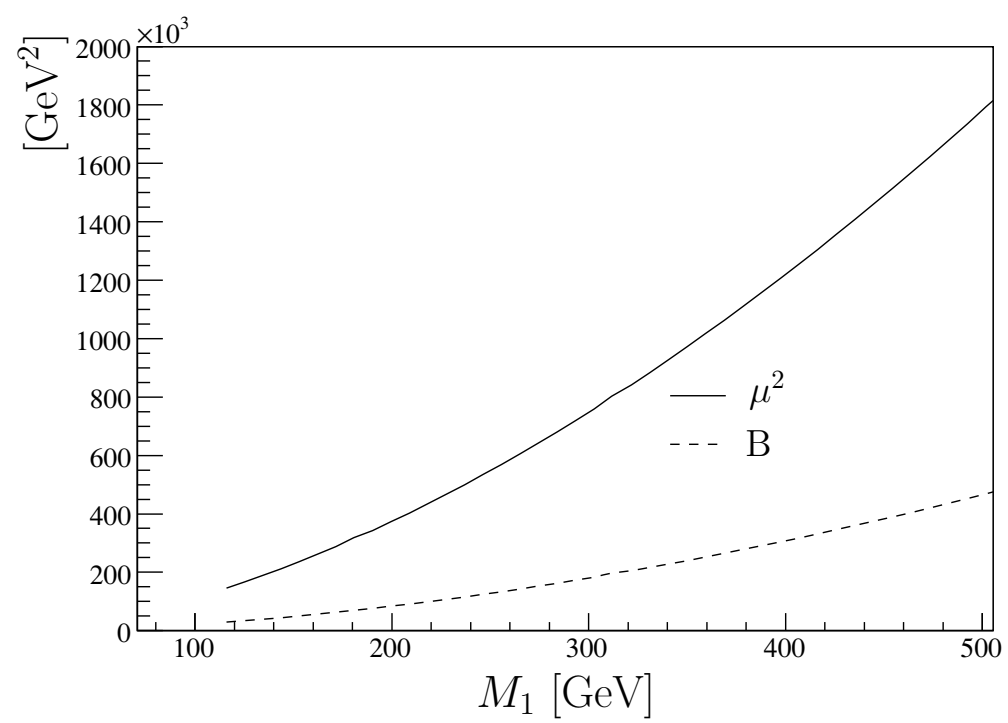

Figure 1. The parameters $\mu$ and $B$ of eqs. (2.3) and (2.4), respectively, as functions of the bino mass $M_{1}$. The plot has been obtained by means of SOFTSUSY3.0 [28].

the mass eigenstate basis:

$$
\begin{aligned}
J_{Z \mu}= & \bar{\chi}_{a}^{0} \gamma_{\mu} V_{a b}^{\left(\chi^{0}\right)} \chi_{b}^{0}+\bar{\chi}_{\alpha}^{-} \gamma_{\mu} V_{\alpha \beta}^{\left(\chi^{-}\right)} \chi_{\beta}^{-}+\bar{\chi}_{\alpha}^{+} \gamma_{\mu} V_{\alpha \beta}^{\left(\chi^{+}\right)} \chi_{\beta}^{+}+\bar{\nu}_{i} \gamma_{\mu} V_{i j}^{(\nu)} \nu_{i}+\bar{e}_{i} \gamma_{\mu} V_{i j}^{(e)} e_{i} \\
& +\left(\bar{\chi}_{a}^{0} \gamma_{\mu} V_{a i}^{(\chi, \nu)} \nu_{i}+\bar{\chi}_{\alpha}^{-} \gamma_{\mu} V_{\alpha i}^{\left(\chi^{-}, e\right)} e_{i}+\bar{\chi}_{\alpha}^{+} \gamma_{\mu} V_{\alpha i}^{\left(\chi^{+}, e^{c}\right)} e_{i}^{c}+\text { h.c. }\right), \\
J_{\mu}^{-}= & \bar{\chi}_{a}^{0} \gamma_{\mu} V_{a \alpha}^{(\chi)} \chi_{\alpha}^{-}+\bar{\chi}_{a}^{0} \gamma_{\mu} V_{a i}^{(\chi, e)} e_{i}+\bar{\nu}_{i} \gamma_{\mu} V_{i \alpha}^{(\nu, \chi)} \chi_{\alpha}^{-}+\bar{\nu}_{i} \gamma_{\mu} V_{i j}^{(\nu, e)} e_{j}, \\
S^{\mu}= & \frac{i}{4}\left[\gamma^{\nu}, \gamma^{\rho}\right] \gamma^{\mu}\left(U_{a}^{(\widetilde{\gamma}, \chi)} \chi_{a}^{0}+U_{a}^{(\widetilde{\gamma}, \chi) *} \chi_{a}^{0 c}+U_{i}^{((\widetilde{\gamma}, \nu)} \nu_{i}+U_{i}^{(\widetilde{\gamma}, \nu) *} \nu_{i}^{c}\right) F_{\nu \rho}+\ldots,
\end{aligned}
$$

where we have defined the photino matrix elements

$$
U_{a}^{(\widetilde{\gamma}, \chi)}=c_{w} U_{a}^{(b, \chi)}+s_{w} U_{a}^{(w, \chi)}, \quad U_{i}^{(\widetilde{\gamma}, \nu)}=c_{w} U_{i}^{(b, \nu)}+s_{w} U_{i}^{(w, \nu)} .
$$

In the appendix the unitary transformations between gauge and mass eigenstates and the resulting matrix elements of neutral and charged currents are given to next-to-leading order in $m_{Z} / \mu$. As we shall see, that expansion converges remarkably well.

In the next section we shall need the couplings of the lightest neutralino $\chi_{1}^{0}$ to charged leptons and neutrinos, and the coupling of the gravitino to photon and neutrino. From the formulae in appendix A one easily obtains ${ }^{4}\left(s_{2 \beta}=2 s_{\beta} c_{\beta}\right)$

$$
\begin{aligned}
V_{1 i}^{(\chi, \nu)} & =-\zeta_{i} \frac{m_{Z} s_{w}}{2 M_{1}}\left(1+\mathcal{O}\left(s_{2 \beta} \frac{m_{Z}^{2}}{\mu^{2}}\right)\right), \\
V_{1 i}^{(\chi, e)} & =-\zeta_{i} \frac{m_{Z} s_{w}}{M_{1}}\left(1+\mathcal{O}\left(s_{2 \beta} \frac{m_{Z}^{2}}{\mu^{2}}\right)\right), \\
U_{i}^{(\tilde{\gamma}, \nu)} & =\zeta_{i} \frac{m_{Z} s_{w} c_{w}\left(M_{2}-M_{1}\right)}{M_{1} M_{2}}\left(1+\mathcal{O}\left(s_{2 \beta} \frac{m_{Z}^{2}}{\mu^{2}}\right)\right) .
\end{aligned}
$$

\footnotetext{
${ }^{4}$ The matrix element $U_{i}^{(\tilde{\gamma}, \nu)}$ agrees with the one used in $[16,36]$ for $M_{2}-M_{1} \ll M_{1}$.
} 
Note that the charged and neutral current couplings agree up to the isospin factor at leading order in $m_{Z}^{2} / \mu^{2}$, i.e., $V_{1 i \mathrm{LO}}^{(\chi, \nu)}=V_{1 i \mathrm{LO}}^{(\chi, e)} / 2$. The mass of the lightest neutralino is given by

$$
m_{\chi_{1}^{0}}=M_{1}-\frac{m_{Z}^{2}\left(M_{1}+\mu s_{2 \beta}\right) s_{w}^{2}}{\mu^{2}-M_{1}^{2}}\left(1+\mathcal{O}\left(\frac{m_{Z}^{2}}{\mu^{2}}\right)\right) .
$$

We have numerically checked that varying $M_{1}$ between 120 and $500 \mathrm{GeV}$, the relative corrections in eqs. (4.15)-(4.18) are less than $10 \%$.

\section{Fermi-LAT and the LHC}

We are now ready to evaluate the implications of recent Fermi-LAT data [21, 22] and cosmological constraints [29-32] for signatures of decaying dark matter at the LHC. We shall first discuss monochromatic gamma-rays produced by gravitino decays and then analyze the implications for a neutralino and a $\widetilde{\tau}$-NLSP, respectively.

In order to keep our analysis transparent we shall not study the most general parameter space of softly broken supersymmetry, but only consider two typical boundary conditions for the supersymmetry breaking parameters of the MSSM at the grand unification scale,

$$
\text { (A) } m_{0}=m_{1 / 2}, \quad a_{0}=0, \quad \tan \beta=10,
$$

with equal universal scalar and gaugino masses, $m_{0}$ and $m_{1 / 2}$, respectively; in this case a bino-like neutralino is the NLSP. The second boundary condition corresponds to no-scale models or gaugino mediation,

$$
\text { (B) } m_{0}=0, \quad m_{1 / 2}, \quad a_{0}=0, \quad \tan \beta=10,
$$

which yields the right-handed stau as NLSP. In both cases, the trilinear scalar coupling $a_{0}$ is put to zero for simplicity. Choosing $\tan \beta=10$ as a representative value of the Higgs vacuum expectation values, only the gaugino mass parameter $m_{1 / 2}$ remains as independent variable; the mass parameters $\mu$ and $B$ are determined by requiring radiative electroweak symmetry breaking with the chosen ratio $\tan \beta$. For both boundary conditions (5.1) and (5.2), the gaugino masses at the electroweak scale satisfy the familiar relations

$$
\frac{M_{3}}{M_{1}} \simeq 6.0, \quad \frac{M_{2}}{M_{1}} \simeq 1.9
$$

For the chosen supergravity models, consistency with electroweak precision tests, gravitino dark matter (GDM) and thermal leptogenesis leads to the following allowed mass ranges of gravitino and lightest neutralino [23],

$$
10 \mathrm{GeV}<m_{3 / 2}<500 \mathrm{GeV}, \quad 100 \mathrm{GeV}<m_{\chi_{1}^{0}}<500 \mathrm{GeV},
$$

where we have used $m_{\chi_{1}^{0}} \simeq M_{1}$ (cf. (4.18)). Note that the masses $M_{1}$ and $m_{3 / 2}$ cannot be chosen independently. The GDM constraint implies that for a given gravitino mass the maximal bino mass is $M_{1}^{\max } \simeq 270 \mathrm{GeV}\left(m_{3 / 2} / 100 \mathrm{GeV}\right)^{1 / 2}[23]$. 


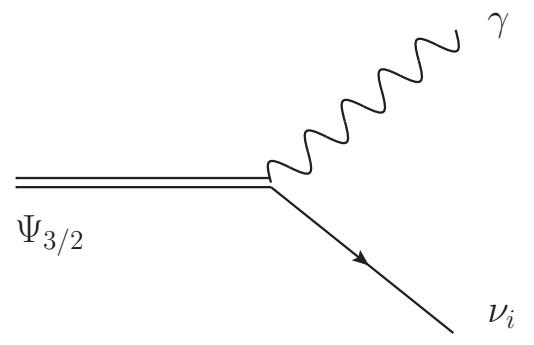

Figure 2. Gravitino decay into photon and neutrino.

Consider now the rate for gravitino decay into photon and neutrino ${ }^{5}$ [13] (cf. figure 2),

$$
\Gamma_{3 / 2}(\gamma \nu)=\frac{1}{32 \pi} \sum_{i}\left|U_{i}^{(\widetilde{\gamma}, \nu)}\right|^{2} \frac{m_{3 / 2}^{3}}{M_{\mathrm{P}}^{2}},
$$

Inserting the matrix element (4.17) one obtains the gravitino lifetime

$$
\tau_{3 / 2}(\gamma \nu)=\frac{32 \sqrt{2}}{\alpha \zeta^{2}} \frac{G_{F} M_{P}^{2}}{m_{3 / 2}^{3}} \frac{M_{1}^{2} M_{2}^{2}}{\left(M_{2}-M_{1}\right)^{2}}\left(1+\mathcal{O}\left(s_{2 \beta} \frac{m_{Z}^{2}}{\mu^{2}}\right)\right)
$$

where $\alpha$ is the electromagnetic fine-structure constant, and we have defined

$$
\zeta^{2}=\sum_{i} \zeta_{i}^{2}
$$

The corrections to the leading order expression in (5.6) are less than $10 \%$. Using eq. (5.3) and $M_{\mathrm{P}}=2.4 \times 10^{18} \mathrm{GeV}$, one obtains

$$
\tau_{3 / 2}(\gamma \nu)=1 \times 10^{27} \mathrm{~s}\left(\frac{\zeta}{10^{-7}}\right)^{-2}\left(\frac{M_{1}}{100 \mathrm{GeV}}\right)^{2}\left(\frac{m_{3 / 2}}{10 \mathrm{GeV}}\right)^{-3} .
$$

Recent Fermi-LAT data yield for dark matter decaying into 2 photons the lower bound on the lifetime $\tau_{\mathrm{DM}}(\gamma \gamma) \gtrsim 1 \times 10^{29} \mathrm{~s}$, which holds for photon energies in the range $30 \mathrm{GeV}<$ $E_{\gamma}<200 \mathrm{GeV}$ [21]. For gravitino decays into photon and neutrino this implies

$$
\tau_{3 / 2}(\gamma \nu) \gtrsim 5 \times 10^{28} \mathrm{~s}, \quad 30 \mathrm{GeV}<E_{\gamma}<200 \mathrm{GeV} .
$$

Since according to the GDM constraint the largest allowed bino mass scales like $M_{1}^{\max } \propto$ $m_{3 / 2}^{1 / 2}$, the largest lifetime (5.8), and therefore the most conservative bound on $\zeta$, is obtained for the smallest value of $m_{3 / 2}$. For small gravitino masses, a rough lower bound on the lifetime can be obtained from the isotropic diffuse gamma-ray flux. The recent Fermi-LAT data give $E^{2} d J /\left.d E\right|_{5 \mathrm{GeV}} \simeq 3 \times 10^{-7} \mathrm{GeV}\left(\mathrm{cm}^{2} \mathrm{sstr}\right)^{-1}$ [22]. From the analysis in [15] one then obtains $\tau_{3 / 2} \gtrsim 10^{28} \mathrm{~s}^{6}$ Together with eq. (5.8) one then obtains the approximate upper bound on the R-parity breaking parameter

$$
\zeta \lesssim 3 \times 10^{-8} .
$$

\footnotetext{
${ }^{5} \Gamma_{3 / 2}(\gamma \nu)$ denotes the sum of the decay rates into photon neutrino and photon antineutrino.

${ }^{6}$ This lifetime is obtained by rescaling in figure 2 of [15] the signal by the factor 0.1 .
} 

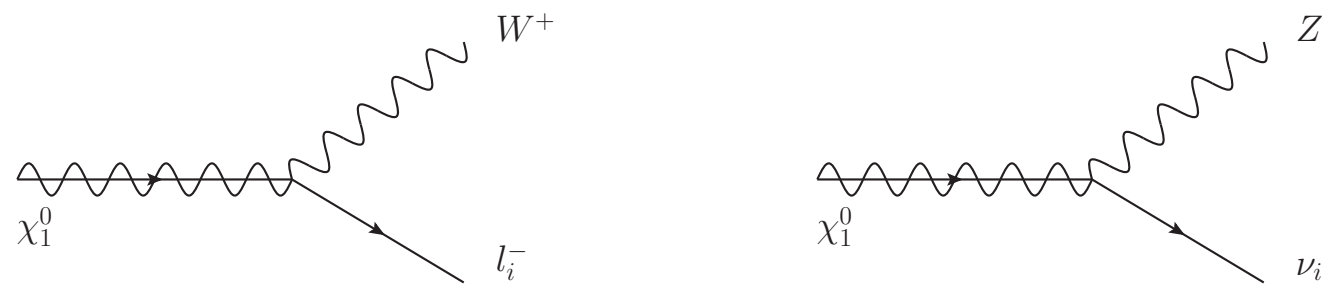

Figure 3. Neutralino decays into charged lepton and W-boson, and neutrino and Z-boson.

On the other hand, the observation of a photon line corresponding to a gravitino lifetime close to the present bound would determine the parameter $\zeta$ as $^{7}$

$$
\zeta_{\text {obs }}=10^{-9}\left(\frac{5 \times 10^{28} \mathrm{~s}}{\tau_{3 / 2}(\gamma \nu)}\right)^{1 / 2}\left(\frac{M_{1}}{200 \mathrm{GeV}}\right)\left(\frac{m_{3 / 2}}{100 \mathrm{GeV}}\right)^{-3 / 2} .
$$

Note the strong dependence of $\zeta_{\text {obs }}$ on the gravitino mass. In (5.11) we have normalized these masses to central values suggested by thermal leptogenesis, electroweak precision tests and gravitino dark matter [23].

\subsection{Neutralino NLSP}

A neutralino NLSP heavier than $100 \mathrm{GeV}$ dominantly decays into charged lepton and Wboson or neutrino and Z-boson [37,38] (cf. figure 3). The partial decay widths are given by

$$
\begin{aligned}
\Gamma\left(\chi_{1}^{0} \rightarrow W^{ \pm} l^{\mp}\right) & =\frac{G_{F} m_{\chi_{1}^{0}}^{3}}{4 \sqrt{2} \pi} \sum_{i}\left|V_{1 i \mathrm{LO}}^{(\chi, e)}\right|^{2} f_{W}\left(m_{\chi_{1}^{0}}\right)\left(1+\mathcal{O}\left(s_{2 \beta} \frac{m_{Z}^{2}}{\mu^{2}}\right)\right), \\
\Gamma\left(\chi_{1}^{0} \rightarrow Z \nu\right) & =\frac{G_{F} m_{\chi_{1}^{0}}^{3}}{2 \sqrt{2} \pi} \sum_{i}\left|V_{1 i \mathrm{LO}}^{(\chi, \nu)}\right|^{2} f_{Z}\left(m_{\chi_{1}^{0}}\right)\left(1+\mathcal{O}\left(s_{2 \beta} \frac{m_{Z}^{2}}{\mu^{2}}\right)\right) .
\end{aligned}
$$

Here $V_{1 i \mathrm{LO}}^{(\chi, e)}$ and $V_{1 i \mathrm{LO}}^{(\chi, \nu)}$ are the charged and neutral current matrix elements at leading order, which are given in eqs. (4.16) and (4.15), respectively, and

$$
f_{W, Z}\left(m_{\chi_{1}^{0}}\right)=\left(1-\frac{m_{W, Z}^{2}}{m_{\chi_{1}^{0}}^{2}}\right)^{2}\left(1+2 \frac{m_{W, Z}^{2}}{m_{\chi_{1}^{0}}^{2}}\right)
$$

is a phase space factor which becomes important for neutralino masses close to the lower bound for $m_{\chi_{1}^{0}}$ of $100 \mathrm{GeV}$ (cf. figure 4).

The total neutralino NLSP width is the sum

$$
\Gamma_{\chi_{1}^{0}}=\Gamma\left(\chi_{1}^{0} \rightarrow W^{ \pm} l^{\mp}\right)+\Gamma\left(\chi_{1}^{0} \rightarrow Z \nu\right) .
$$

Using the matrix elements (4.15) and (4.16), one obtains the branching ratios

$$
B R\left(\chi_{1}^{0} \rightarrow W^{ \pm} l^{\mp}\right) \simeq 2 B R\left(\chi_{1}^{0} \rightarrow Z \nu\right) .
$$

\footnotetext{
${ }^{7}$ The results (5.10) and (5.11) are approximately consistent with the recent analysis [20].
} 


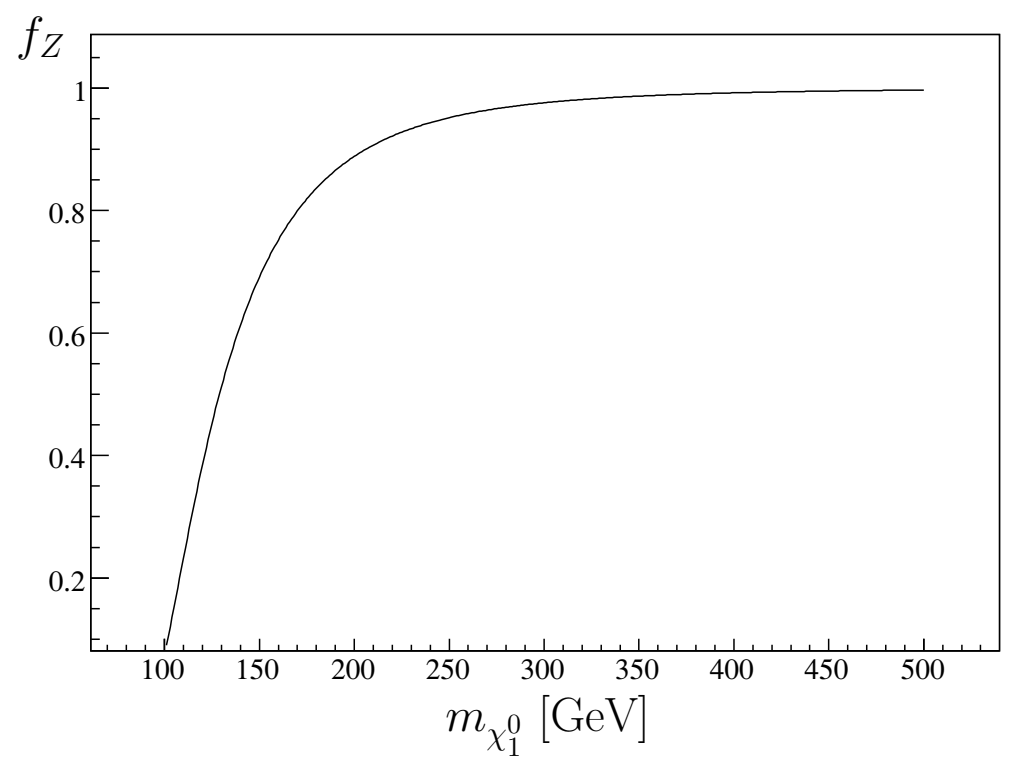

Figure 4. Phase space suppression factor for neutralino decay to $Z$-boson and neutrino.

Furthermore, the flavour structure of our model implies

$$
B R\left(\chi_{1}^{0} \rightarrow W^{ \pm} \mu^{\mp}\right) \simeq B R\left(\chi_{1}^{0} \rightarrow W^{ \pm} \tau^{\mp}\right) .
$$

Using the matrix elements (4.15), (4.16) and (4.17) for neutral, charged and supercurrent, respectively, one can express the neutralino lifetime directly in terms of the gravitino lifetime,

$$
\begin{aligned}
\tau_{\chi_{1}^{0}}= & \frac{c_{w}^{2}}{2 \sqrt{2}} \frac{\left(M_{2}-M_{1}\right)^{2}}{M_{2}^{2}} \frac{m_{3 / 2}^{3}}{G_{F} M_{P}^{2} m_{\chi_{1}^{0}}^{3}} \tau_{3 / 2}(\gamma \nu) \\
& \times\left(2 f_{W}\left(m_{\chi_{1}^{0}}\right)+f_{Z}\left(m_{\chi_{1}^{0}}\right)\right)^{-1}\left(1+\mathcal{O}\left(s_{2 \beta} \frac{m_{Z}^{2}}{\mu^{2}}\right)\right) .
\end{aligned}
$$

With the mass relations (4.18) and (5.3) one then obtains for the minimal neutralino decay length

$$
\begin{aligned}
c \tau_{\chi_{1}^{0}} \gtrsim 80 \mathrm{~cm} & \left(\frac{m_{\chi_{1}^{0}}}{150 \mathrm{GeV}}\right)^{-3}\left(\frac{m_{3 / 2}}{10 \mathrm{GeV}}\right)^{3}\left(\frac{\tau_{3 / 2}(\gamma \nu)}{1 \times 10^{28} \mathrm{~s}}\right) \\
& \times\left(2 f_{W}\left(m_{\chi_{1}^{0}}\right)+f_{Z}\left(m_{\chi_{1}^{0}}\right)\right)^{-1}\left(1+\mathcal{O}\left(s_{2 \beta} \frac{m_{Z}^{2}}{\mu^{2}}\right)\right) .
\end{aligned}
$$

In eqs. (5.18) and (5.19) the corrections to the leading order expressions are less than $10 \%$. We emphasize again the strong dependence of this lower bound on the neutralino and gravitino masses. For instance, for a gravitino mass of $100 \mathrm{GeV}$ and the Fermi-LAT bound $\tau_{3 / 2} \gtrsim 5 \times 10^{28} \mathrm{~s}$, which applies for gravitino masses in the range $60 \mathrm{GeV}<m_{3 / 2}<400 \mathrm{GeV}$, one obtains $c \tau_{\chi_{1}^{0}} \simeq 2 \mathrm{~km}$ for a neutralino mass of $150 \mathrm{GeV}$. It is very interesting that such neutralino lifetimes are detectable at the LHC [39]. 
We conclude that, given the current bounds on the gravitino lifetime, a neutralino NLSP may still decay into gauge boson and lepton inside the detector, yielding a spectacular signature. However, for most of the parameter space a neutralino NLSP decays outside the detector, leading to events indistinguishable from ordinary neutralino dark matter.

\section{$5.2 \quad \widetilde{\tau}$-Lepton NLSP}

Contrary to the neutralino NLSP decay, the R-parity violating decays of a $\widetilde{\tau}_{1}$-NLSP strongly depend on the flavour structure and the supersymmetry breaking parameters. The relative strength of the various decay modes becomes most transparent in the field basis where all bilinear R-parity breaking terms vanish, as discussed in section 2. Since the R-parity breaking Yukawa couplings are proportional to the ordinary Yukawa couplings, decays into fermions of the second and third generation dominate. The leading partial decay widths of left- and right-handed $\widetilde{\tau}$-leptons are (cf. (2.14))

$$
\begin{aligned}
\Gamma_{\widetilde{\tau}_{L}}\left(\tau_{R} \nu\right) & =\frac{1}{16 \pi} \sum_{i}\left|\hat{\lambda}_{i 33}\right|^{2} m_{\widetilde{\tau}_{L}}, \\
\Gamma_{\widetilde{\tau}_{L}}\left(\bar{t}_{L} b_{R}\right) & =\Gamma_{\widetilde{\tau}_{L}}\left(\bar{t}_{L} s_{R}\right)=\frac{3}{16 \pi}\left|\lambda_{333}^{\prime}\right|^{2} m_{\widetilde{\tau}_{L}}, \\
\Gamma_{\widetilde{\tau}_{L}}\left(\bar{t}_{R} b_{L}\right) & =\frac{3}{16 \pi}\left|\hat{\lambda}_{333}^{\prime}\right|^{2} m_{\widetilde{\tau}_{L}}, \\
\Gamma_{\widetilde{\tau}_{R}}\left(\tau_{L} \nu\right) & =\Gamma_{\widetilde{\tau}_{R}}\left(\mu_{L} \nu\right)=\frac{1}{16 \pi} \sum_{i}\left|\lambda_{i 33}\right|^{2} m_{\widetilde{\tau}_{R}} .
\end{aligned}
$$

In the flavour model discussed in section 3 , the order of magnitude of the various decay widths is determined by the power of the hierarchy parameter $\eta\left(\eta^{2} \simeq 1 / 300\right)$,

$$
\begin{aligned}
\Gamma_{\widetilde{\tau}_{L}}\left(\tau_{R} \nu\right) & \sim \Gamma_{\widetilde{\tau}_{R}}\left(\tau_{L} \nu\right)=\Gamma_{\widetilde{\tau}_{R}}\left(\mu_{L} \nu\right) \\
& \sim \Gamma_{\widetilde{\tau}_{L}}\left(\bar{t}_{L} b_{R}\right) \sim \Gamma_{\widetilde{\tau}_{L}}\left(\bar{t}_{L} s_{R}\right) \sim \eta^{4} \Theta^{2} m_{\widetilde{\tau}}, \\
\Gamma_{\widetilde{\tau}_{L}}\left(\bar{t}_{R} b_{L}\right) & \sim \eta^{2} \Theta^{2} m_{\widetilde{\tau}} .
\end{aligned}
$$

The lightest mass eigenstate $\widetilde{\tau}_{1}$ is a linear combination of $\widetilde{\tau}_{L}$ and $\widetilde{\tau}_{R}$,

$$
\widetilde{\tau}_{1}=\sin \theta_{\tau} \widetilde{\tau}_{L}+\cos \theta_{\tau} \widetilde{\tau}_{R}
$$

From the above equations one obtains the $\widetilde{\tau}_{1}$-decay width

$$
\Gamma_{\widetilde{\tau}_{1}}=\sin ^{2} \theta_{\tau}\left(\Gamma_{\widetilde{\tau}_{L}}\left(\tau_{R} \nu\right)+2 \Gamma_{\widetilde{\tau}_{L}}\left(\bar{t}_{L} b_{R}\right)+\Gamma_{\widetilde{\tau}_{L}}\left(\bar{t}_{R} b_{L}\right)\right)+2 \cos ^{2} \theta_{\tau} \Gamma_{\widetilde{\tau}_{R}}\left(\tau_{L} \nu\right) .
$$

The total width is dominated by the contributions $\widetilde{\tau}_{R} \rightarrow \tau_{L} \nu, \mu_{L} \nu$ and $\widetilde{\tau}_{L} \rightarrow \bar{t}_{R} b_{L}$, respectively,

$$
\Gamma_{\widetilde{\tau}_{1}}=\sin ^{2} \theta_{\tau} \Gamma_{\widetilde{\tau}_{L}}\left(\bar{t}_{R} b_{L}\right)+2 \cos ^{2} \theta_{\tau} \Gamma_{\widetilde{\tau}_{R}}\left(\tau_{L} \nu\right),
$$

and it can be directly expressed in terms of the $\tau$-lepton and top-quark masses,

$$
\Gamma_{\widetilde{\tau}_{1}}=\frac{\epsilon^{2}}{16 \pi v^{2}}\left(3 m_{t}^{2} \sin ^{2} \theta_{\tau}+2 m_{\tau}^{2} \tan ^{2} \beta \cos ^{2} \theta_{\tau}\right) m_{\widetilde{\tau}_{1}},
$$




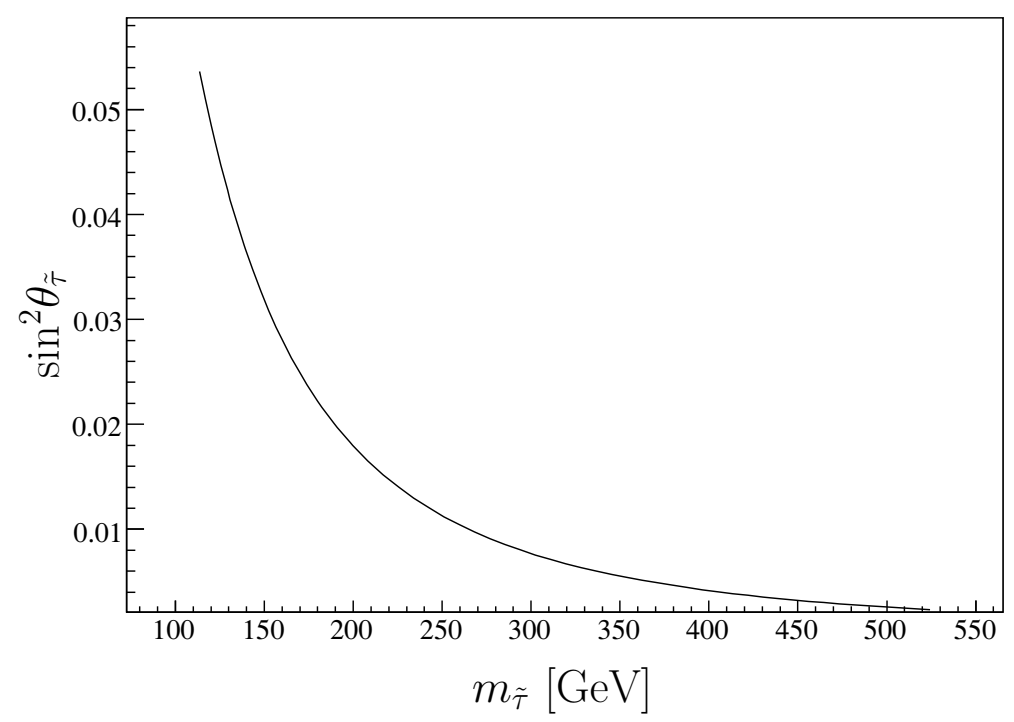

Figure 5. $\widetilde{\tau}$-mixing angle: $\sin ^{2}\left(\theta_{\tau}\right)$ as function of the lightest $\widetilde{\tau}$-mass $m_{\widetilde{\tau}_{1}}$.

where we have assumed

$$
\epsilon_{2,3}=\epsilon_{2,3}^{\prime}=\epsilon_{2,3}^{\prime \prime} \equiv \epsilon
$$

This corresponds to the parameter choice $a=b=c=1$ in eq. (3.14). Note that $\widetilde{\tau}_{1}$-decay width and branching ratios have a considerable uncertainty since these parameters depend on the unspecified mechanism of supersymmetry breaking. From eqs. (2.18), (3.6) and $\eta \simeq 0.06$, one obtains for the R-parity breaking parameter

$$
\epsilon \simeq \zeta \simeq \eta \Theta \simeq 6 \times 10^{-8}
$$

which is consistent with the present upper bound (5.10) within the theoretical uncertainties.

The dependence of the mixing angle $\theta_{\tau}$ on $m_{\widetilde{\tau}_{1}}$ is shown in figure 5 for the boundary condition (5.2). For masses below the top-bottom threshold only leptonic $\widetilde{\tau}_{1}$-decays are possible. When the decay into top-bottom pairs becomes kinematically allowed, $\sin ^{2} \theta_{\tau}$ is small. However, the suppression by a small mixing angle is compensated by the larger Yukawa coupling compared to the leptonic decay mode. This is a direct consequence of the couplings $\hat{\lambda}^{\prime}$ which were not taken into account in previous analyses.

Due to the competition between mixing angle suppression and hierarchical Yukawa couplings, the top-bottom threshold is clearly visible in the $\widetilde{\tau}_{1}$-decay length as well as the branching ratios into leptons and heavy quarks. This is illustrated in figures 6 and 7 , respectively, where these observables are plotted as functions of $m_{\widetilde{\tau}_{1}}$. Representative values of the $\widetilde{\tau}_{1}$-decay lengths below and above the top-bottom threshold are

$$
\begin{aligned}
& m_{\widetilde{\tau}_{1}}<m_{t}+m_{b}:\left.\quad c \tau_{\widetilde{\tau}_{1}}\right|_{150 \mathrm{GeV}}=1.4 \mathrm{~m}\left(\frac{\epsilon}{5 \times 10^{-8}}\right)^{-2}, \\
& m_{\widetilde{\tau}_{1}}>m_{t}+m_{b}:\left.\quad c \tau_{\widetilde{\tau}_{1}}\right|_{250 \mathrm{GeV}}=0.6 \mathrm{~m}\left(\frac{\epsilon}{5 \times 10^{-8}}\right)^{-2} .
\end{aligned}
$$




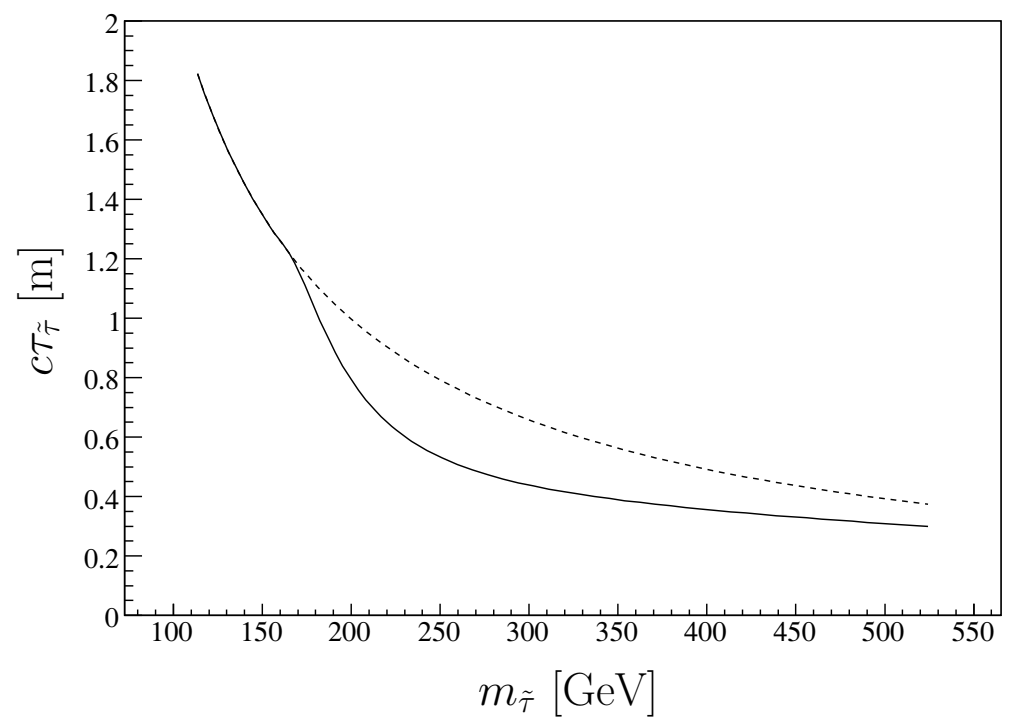

Figure 6. $\widetilde{\tau}_{1}$-decay length as function of $m_{\widetilde{\tau}_{1}}$. Above the top-bottom threshold hadronic decays decrease the $\widetilde{\tau}_{1}$-lifetime.

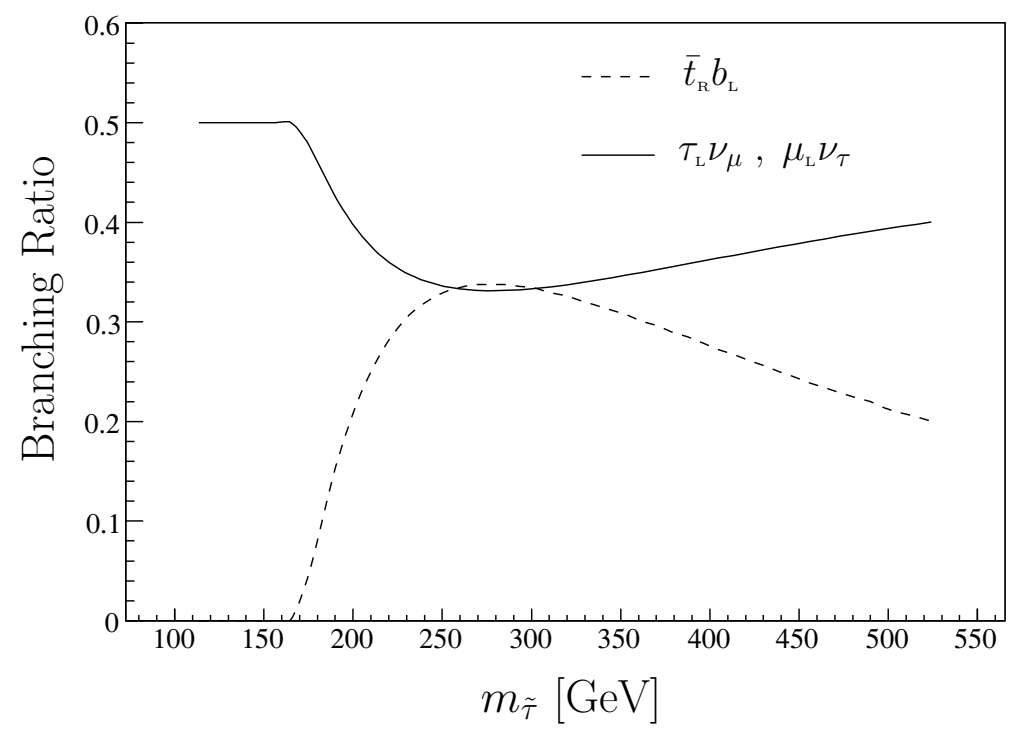

Figure 7. $\widetilde{\tau}_{1}$-branching ratios as functions of $m_{\widetilde{\tau}_{1}}$. The dependence on the $\widetilde{\tau}_{1}$-mass is determined by the top-bottom threshold and the mass dependence of the $\widetilde{\tau}_{1}$-mixing angle.

Choosing for $\epsilon$ the representative value (5.11) from gravitino decay, $\epsilon=\zeta_{\text {obs }}=10^{-9}$, one obtains $c \tau_{\widetilde{\tau}_{1}}=4 \mathrm{~km}(1 \mathrm{~km})$ for $m_{\widetilde{\tau}_{1}}=150 \mathrm{GeV}(250 \mathrm{GeV})$. It is remarkable that such lifetimes can be measured at the LHC [39, 40].

Is it possible to avoid the severe constraint from gravitino decays on the $\widetilde{\tau}_{1}$-decay length? In principle, both observables are independent, and the unknown constants in the definition of $\epsilon, \epsilon^{\prime}$ and $\epsilon^{\prime \prime}$ can be adjusted such that $\zeta=0$. However, this corresponds to a strong fine-tuning, unrelated to an underlying symmetry. To illustrate this, consider the 
case where the soft R-parity breaking parameters vanish at the GUT scale, $B_{i}=m_{i d}^{2}=0$, which was discussed in section 3. In bilinear R-parity breaking, also the R-parity violating Yukawa couplings vanish at the GUT scale. With the one-loop radiative corrections at the electroweak scale (cf. (3.19); $\epsilon_{i}=\mu_{i} / \mu$ ),

$$
B_{i}\left(\Lambda_{\mathrm{EW}}\right)=\frac{\epsilon_{i} \mu}{16 \pi^{2}}\left(\frac{6}{5} g^{\prime 2} M_{1}+6 g^{2} M_{2}\right) \ln \frac{\Lambda_{\mathrm{GUT}}}{\Lambda_{\mathrm{EW}}}, \quad m_{i d}^{2}\left(\Lambda_{\mathrm{EW}}\right)=0,
$$

and $M_{1,2} \sim \mu$, one reads off from eqs. (2.10), (2.12) and (2.13)

$$
\epsilon_{i}^{\prime}, \epsilon_{i}^{\prime \prime}=\mathcal{O}\left(\epsilon_{i}\right) .
$$

Hence, all R-parity breaking parameters are naturally of the same order, unless the finetuning also includes radiative corrections between the GUT scale and the electroweak scale.

Even if one accepts the fine-tuning $\zeta=0$, one still has to satisfy the cosmological bounds on R-parity violating couplings, which yield $\epsilon_{i}=\mu_{i} / \mu \lesssim 10^{-6}$ [32]. In the flavour model discussed in section 3 this corresponds to the choice $a=20$ in eq. (3.13). For the smaller $\widetilde{\tau}_{1}$-mass, which is preferred by electroweak precision tests, one then obtains the lower bound on the decay length

$$
\left.c \tau_{\widetilde{\tau}_{1}}\right|_{150 \mathrm{GeV}} \gtrsim 4 \mathrm{~mm} .
$$

However, let us emphasize again that current constraints from Fermi-LAT on the diffuse gamma-ray spectrum indicate decay lengths several orders of magnitude larger.

\subsection{Planck mass measurement}

It has been pointed out in [12] that, in principle, one can determine the Planck mass from decay properties of a $\widetilde{\tau}$-NLSP together with the observation of a photon line in the diffuse gamma-ray flux, which is produced by gravitino decays. This is similar to the proposed microscopic determination of the Planck mass based on decays of very long lived $\widetilde{\tau}$-NLSP's in the case of a stable gravitino [41].

From our analysis of NLSP decays in this section it is clear that neutralino NLSP decays are particularly well suited for a measurement of the Planck mass, which does not require any additional assumptions. Eq. (5.18) implies $\left(G_{F}=\sqrt{2} /\left(4 v^{2}\right)\right)$,

$$
\begin{aligned}
M_{\mathrm{P}}= & c_{w} v \frac{M_{2}-M_{1}}{M_{2}}\left(\frac{m_{3 / 2}}{m_{\chi_{1}^{0}}}\right)^{3 / 2}\left(\frac{\tau_{3 / 2}(\gamma \nu)}{\tau_{\chi_{1}^{0}}}\right)^{1 / 2} \\
& \times\left(2 f_{W}\left(m_{\chi_{1}^{0}}\right)+f_{Z}\left(m_{\chi_{1}^{0}}\right)\right)^{-1 / 2}\left(1+\mathcal{O}\left(s_{2 \beta} \frac{m_{Z}^{2}}{\mu^{2}}\right)\right) .
\end{aligned}
$$

As expected, for gravitino and neutralino masses of the same order of magnitude, the ratio of the two-body lifetimes is determined by the ratio of the electroweak scale and the Planck mass,

$$
\frac{\tau_{\chi_{1}^{0}}}{\tau_{3 / 2}(\gamma \nu)} \sim \frac{v^{2}}{M_{\mathrm{P}}^{2}}
$$


Quantitatively, using the relation (5.3) for the gaugino masses, one finally obtains $(v=$ $174 \mathrm{GeV})$,

$$
\begin{aligned}
M_{\mathrm{P}}= & 3.6 \times 10^{18} \mathrm{GeV}\left(\frac{m_{3 / 2}}{m_{\chi_{1}^{0}}}\right)^{3 / 2}\left(\frac{\tau_{3 / 2}(\gamma \nu)}{10^{28} \mathrm{~s}}\right)^{1 / 2}\left(\frac{\tau_{\chi_{1}^{0}}}{10^{-7} \mathrm{~s}}\right)^{-1 / 2} \\
& \times\left(2 f_{W}\left(m_{\chi_{1}^{0}}\right)+f_{Z}\left(m_{\chi_{1}^{0}}\right)\right)^{-1 / 2}\left(1+\mathcal{O}\left(s_{2 \beta} \frac{m_{Z}^{2}}{\mu^{2}}\right)\right) .
\end{aligned}
$$

It is remarkable that the observation of a photon line in the diffuse gamma-ray flux, together with a measurement of the neutralino lifetime at the LHC, can provide a microscopic determination of the Planck mass.

\section{Summary and conclusions}

We have studied a supersymmetric extension of the Standard Model with small R-parity breaking related to spontaneous $B-L$ breaking, which is consistent with primordial nucleosynthesis, thermal leptogenesis and gravitino dark matter. We have considered supergravity models with universal boundary conditions at the GUT scale, which lead to scalar tau or bino-like neutralino as NLSP. Supersymmetry breaking terms have been introduced by means of higher-dimensional operators. The size of the soft terms corresponds to gravity or gaugino mediation.

We have analyzed our model, which represents a special case of bilinear R-parity breaking, in a basis of scalar $\mathrm{SU}(2)$ doublets, where all bilinear terms vanish. In this basis one has R-parity violating Yukawa and gaugino couplings. They are given in terms of ordinary Yukawa couplings and 9 R-parity breaking parameters $\epsilon_{i}, \epsilon_{i}^{\prime}$ and $\epsilon_{i}^{\prime \prime}, i=1, \ldots, 3$, which are constrained by the flavour symmetry of the model. The R-parity violating couplings include terms proportional to the up-quark Yukawa couplings, which were not taken into accound in previous analyses.

The main goal of this paper are the quantitative connection between gravitino decays and NLSP decays, and the corresponding implications of recent Fermi-LAT data on the isotropic diffuse gamma-ray flux for superparticle decays at the LHC. To establish this connection one needs the relevant R-parity breaking matrix elements of neutral, charged and supercurrents. For the considered supergravity models these matrix elements can be obtained analytically to good approximation, since the diagonalization of the neutralinoneutrino and chargino-lepton mass matrices in powers of $m_{Z} / \mu$ converges well, as demonstrated in the appendix. The analytic expressions for the decay rates make the implications of the Fermi-LAT data for NLSP decays very transparent.

Our main quantitative results are the branching ratios for NLSP decays and the lower bounds on their decay lengths. For a neutralino NLSP with $m_{\chi_{1}^{0}}=150 \mathrm{GeV}$, the FermiLAT data yield the lower bound $c \tau_{\chi_{1}^{0}} \gtrsim 30 \mathrm{~cm}$. This bound does not depend on details of the superparticle mass spectrum or the flavour structure of the model. It directly follows from the comparison of two-particle gravitino and neutralino decays. On the contrary, there exists no model independent lower bound on the $\widetilde{\tau}_{1}$-decay length. The natural relation 
between gravitino and $\widetilde{\tau}$-decay widths can be avoided by fine-tuning. In this case the cosmological constraint that the baryon asymmetry is not washed out leads to the lower bound $c \tau_{\widetilde{\tau}_{1}} \gtrsim 4 \mathrm{~mm}$.

Without fine-tuning parameters the diffuse gamma-ray flux produced by gravitino decays constrains the lifetime of a neutralino as well as a $\widetilde{\tau}$-NLSP. For typical masses, $m_{3 / 2} \sim 100 \mathrm{GeV}$ and $m_{\mathrm{NLSP}} \sim 150 \mathrm{GeV}$, the discovery of a photon line with an intensity close to the present Fermi-LAT limit would imply a decay length $c \tau_{\mathrm{NLSP}}$ of several hundered meters. This is a definite prediction of a class of supergravity models. It is very interesting that such lifetimes can be measured at the LHC [39, 40].

Finally, it is intriguing that the observation of a photon line in the diffuse gammaray flux, together with a measurement of the neutralino lifetime at the LHC, can yield a microscopic determination of the Planck mass, a crucial test of local supersymmetry.

\section{Acknowledgments}

We would like to thank L. Covi, M. Grefe and C. Weniger for helpful discussions. This work was supported by the German Science Foundation (DFG) within the Collaborative Research Center 676 "Particles, Strings and the Early Universe" and the Hamburg Excellence Initiative "Connecting Particles with the Cosmos".

\section{A Gauge and mass eigenstates}

\section{A.1 Mass matrix diagonalization}

The mass matrices $\mathcal{M}^{N}$ and $\mathcal{M}^{C}$ in the gauge eigenbasis were explicitly given in eqs. (4.7) and (4.8), respectively,

$$
\begin{aligned}
\mathcal{M}^{N}= & \left(\begin{array}{ccccc}
M_{1} & 0 & m_{Z} s_{\beta} s_{w} & -m_{Z} c_{\beta} s_{w} & -\zeta_{i} m_{Z} s_{w} \\
0 & M_{2} & -m_{Z} s_{\beta} c_{w} & m_{Z} c_{\beta} c_{w} & \zeta_{i} m_{Z} c_{w} \\
m_{Z} s_{\beta} s_{w} & -m_{Z} s_{\beta} c_{w} & 0 & -\mu & 0 \\
-m_{Z} c_{\beta} s_{w} & m_{Z} c_{\beta} c_{w} & -\mu & 0 & 0 \\
-\zeta_{i} m_{Z} s_{w} & \zeta_{i} m_{Z} c_{w} & 0 & 0 & 0
\end{array}\right), \\
\mathcal{M}^{C} & =\left(\begin{array}{ccccc}
M_{2} & m_{Z} s_{\beta} c_{w} & 0 & 0 & 0 \\
m_{Z} c_{\beta} c_{w} & \mu & \zeta_{1} h_{11}^{e} \mu & \zeta_{2} h_{22}^{e} \mu & \zeta_{3} h_{33}^{e} \mu \\
\zeta_{1} m_{Z} c_{w} & 0 & h_{11}^{e} v c_{\beta} & 0 & 0 \\
\zeta_{2} m_{Z} c_{w} & 0 & 0 & h_{22}^{e} v c_{\beta} & 0 \\
\zeta_{3} m_{Z} c_{w} & 0 & 0 & 0 & h_{33}^{e} v c_{\beta}
\end{array}\right) .
\end{aligned}
$$

For non-vanishing $R$-parity breaking parameters $\zeta_{i}, i=1, \ldots, 3$, they induce a mixing between gauginos, Higgsinos and leptons,

$$
\begin{aligned}
-\mathcal{L} \supset & \frac{1}{2}\left(b, w^{3}, h_{u}^{0}, h_{d}^{0}, \nu_{i}\right) \mathcal{M}^{N}\left(b, w^{3}, h_{u}^{0}, h_{d}^{0}, \nu_{i}\right)^{T} \\
& +\left(\left(w^{-}, h_{d}^{-}, e_{i}\right) \mathcal{M}^{C}\left(w^{+}, h_{u}^{+}, e_{i}^{c}\right)^{T}+\text { h.c. }\right) .
\end{aligned}
$$


The matrices $\mathcal{M}^{N}$ and $\mathcal{M}^{C}$ are diagonalized by unitary and bi-unitary transformations, respectively,

$$
U^{(n) T} \mathcal{M}^{N} U^{(n)}=\mathcal{M}_{\mathrm{diag}}^{N}, \quad U^{(c) \dagger} \mathcal{M}^{C} \widetilde{U}^{(c)}=\mathcal{M}_{\mathrm{diag}}^{C},
$$

where $U^{(n) \dagger} U^{(n)}=U^{(c) \dagger} U^{(c)}=\widetilde{U}^{(c) \dagger} \widetilde{U}^{(c)}=1$. These unitary transformations relate the neutral and charged gauge eigenstates to the mass eigenstates $\left(\chi_{a}^{0}, \nu_{i}^{\prime}\right)(a=1, \ldots, 4)$ and $\left(\chi_{\alpha}^{-}, e_{i}^{\prime}\right),\left(\chi_{\alpha}^{+}, e_{i}^{c^{\prime}}\right)(\alpha=1,2)$, respectively.

In this work we consider the two boundary conditions $(A)$ and $(B)$, defined in eqs. (5.1) and (5.2), respectively. The corresponding supergravity models satisfy the relation (4.9), $m_{Z}<M_{1,2}<\mu$, and in the regime $120 \mathrm{GeV} \lesssim M_{1} \lesssim 500 \mathrm{GeV}$ one finds $0.07 \lesssim m_{Z} / \mu \lesssim$ 0.25. We diagonalized the above mass matrices to first order in the small parameters $\zeta_{i}$ and to second order in $m_{Z} / \mu$. The size of the relative corrections given below has been calculated for the above parameter range using SOFTSUSY3.0 [28]. As we shall see, the relative corrections are of order $m_{Z}^{2} / \mu^{2}$, and the expansion converges well for most matrix elements.

The neutralino and neutrino mass eigenvalues are

$$
\begin{aligned}
& m_{\chi_{1}^{0}}=M_{1}-\frac{m_{Z}^{2}\left(M_{1}+\mu s_{2 \beta}\right) s_{w}^{2}}{\mu^{2}-M_{1}^{2}}\left(1+\mathcal{O}\left(\frac{m_{Z}^{2}}{\mu^{2}}\right)\right), \\
& m_{\chi_{2}^{0}}=M_{2}-\frac{m_{Z}^{2}\left(M_{2}+\mu s_{2 \beta}\right) c_{w}^{2}}{\mu^{2}-M_{2}^{2}}\left(1+\mathcal{O}\left(\frac{m_{Z}^{2}}{\mu^{2}}\right)\right), \\
& m_{\chi_{3}^{0}}=\mu+\frac{m_{Z}^{2}\left(\mu-M_{1} c_{w}^{2}-M_{2} s_{w}^{2}\right)\left(1+s_{2 \beta}\right)}{2\left(\mu-M_{1}\right)\left(\mu-M_{2}\right)}\left(1+\mathcal{O}\left(\frac{m_{Z}^{2}}{\mu^{2}}\right)\right), \\
& m_{\chi_{4}^{0}}=-\mu-\frac{m_{Z}^{2}\left(\mu+M_{1} c_{w}^{2}+M_{2} s_{w}^{2}\right)\left(1-s_{2 \beta}\right)}{2\left(\mu+M_{1}\right)\left(\mu+M_{2}\right)}\left(1+\mathcal{O}\left(s_{2 \beta} \frac{m_{Z}^{2}}{\mu^{2}}\right)\right), \\
& m_{\nu_{i}}=0+\mathcal{O}\left(\zeta^{2} \frac{m_{Z}^{2}}{\mu^{2}}\right) .
\end{aligned}
$$

We checked numerically that relative corrections $\mathcal{O}\left(m_{Z}^{2} / \mu^{2}\right)$ to the above neutralino masses are smaller than $0.05,0.15,0.10,0.001$, for $m_{\chi_{1}^{0}}, \ldots, m_{\chi_{4}^{0}}$, respectively.

The chargino and lepton mass eigenvalues are

$$
\begin{aligned}
& m_{\chi_{1}^{ \pm}}=M_{2}-\frac{m_{Z}^{2}\left(M_{2}+\mu s_{2 \beta}\right) c_{w}^{2}}{2\left(\mu^{2}-M_{2}^{2}\right)}\left(1+\mathcal{O}\left(\frac{m_{Z}^{2}}{\mu^{2}}\right)\right), \\
& m_{\chi_{2}^{ \pm}}=\mu+\frac{m_{Z}^{2}\left(\mu+M_{2} s_{2 \beta}\right) c_{w}^{2}}{2\left(\mu^{2}-M_{2}^{2}\right)}\left(1+\mathcal{O}\left(\frac{m_{Z}^{2}}{\mu^{2}}\right)\right), \\
& m_{e_{i}^{\prime}}=h_{i i}^{e} v c_{\beta}\left(1+\mathcal{O}\left(\zeta^{2} \frac{m_{Z}^{2}}{\mu^{2}}\right)\right) .
\end{aligned}
$$

Here the relative corrections of $\mathcal{O}\left(m_{Z}^{2} / \mu^{2}\right)$ are numerically smaller than $5 \%$.

The unitary matrix $U^{(n)}$ from eq. (A.2) can be written as

$$
U^{(n)}=\left(\begin{array}{c|c}
U_{a b}^{\left(\chi^{0}\right)} & U_{a i}^{\left(\chi^{0}, \nu\right)} \\
\hline U_{i a}^{\left(\nu, \chi^{0}\right)} & U_{i j}^{(\nu)}
\end{array}\right),
$$


with

$$
\begin{aligned}
& U_{a b}^{\left(\chi^{0}\right)}=\left(\begin{array}{llll}
1 & 0 & 0 & 0 \\
0 & 1 & 0 & 0 \\
0 & 0 & -\frac{1}{\sqrt{2}} & \frac{1}{\sqrt{2}} \\
0 & 0 & \frac{1}{\sqrt{2}} & \frac{1}{\sqrt{2}}
\end{array}\right) \\
& +\left(\begin{array}{llll}
-\frac{m_{Z}{ }^{2}\left(M_{1}^{2}+2 \mu s_{2 \beta} M_{1}+\mu^{2}\right) s_{w}^{2}}{2\left(M_{1}^{2}-\mu^{2}\right)^{2}} & \frac{m_{Z}{ }^{2}\left(M_{2}+\mu s_{2 \beta}\right) s_{2 w}}{2\left(M_{1}-M_{2}\right)\left(M_{2}^{2}-\mu^{2}\right)} & \frac{m_{Z}\left(c_{\beta}+s_{\beta}\right) s_{w}}{\sqrt{2}\left(M_{1}-\mu\right)} & \frac{m_{Z}\left(c_{\beta}-s_{\beta}\right) s_{w}}{\sqrt{2}\left(M_{1}+\mu\right)} \\
-\frac{m_{Z}{ }^{2}\left(M_{1}+\mu s_{2 \beta}\right) s_{2 w}}{2\left(M_{1}-M_{2}\right)\left(M_{1}{ }^{2}-\mu^{2}\right)} & -\frac{m_{Z}^{2} c_{w}^{2}\left(M_{2}^{2}+2 \mu s_{2 \beta} M_{2}+\mu^{2}\right)}{2\left(M_{2}{ }^{2}-\mu^{2}\right)^{2}} & -\frac{m_{Z} c_{w}\left(c_{\beta}+s_{\beta}\right)}{\sqrt{2}\left(M_{2}-\mu\right)} & \frac{m_{Z} c_{w}\left(s_{\beta}-c_{\beta}\right)}{\sqrt{2}\left(M_{2}+\mu\right)} \\
\frac{m_{Z}\left(\mu c_{\beta}+M_{1} s_{\beta}\right) s_{w}}{M_{1}^{2}-\mu^{2}} & -\frac{m_{Z} c_{w}\left(\mu c_{\beta}+M_{2} s_{\beta}\right)}{M_{2}^{2}-\mu^{2}} & \frac{m_{Z}{ }^{2}\left(c_{\beta}+s_{\beta}\right)}{\mu^{2}} x_{1} & \frac{\left(c_{\beta}-s_{\beta}\right) m_{Z}}{\mu^{2}} x_{2} \\
-\frac{m_{Z}\left(M_{1} c_{\beta}+\mu s_{\beta}\right) s_{w}}{M_{1}^{2}-\mu^{2}} & \frac{m_{Z} c_{w}\left(M_{2} c_{\beta}+\mu s_{\beta}\right)}{M_{2}{ }^{2}-\mu^{2}} & \frac{\left(c_{\beta}+s_{\beta}\right) m_{Z}{ }^{2}}{\mu^{2}} x_{3} & \frac{\left(c_{\beta}-s_{\beta}\right) m_{Z}{ }^{2}}{\mu^{2}} x_{4}
\end{array}\right) \\
& \times\left(1+\mathcal{O}\left(\frac{m_{Z}^{2}}{\mu^{2}}\right)\right),
\end{aligned}
$$

where we used the abbreviations

$$
\begin{aligned}
& x_{1}=\frac{\mu}{4 \sqrt{2}}\left(\frac{\left(M_{2} s_{\beta}-\left(M_{2}-2 \mu\right) c_{\beta}\right) c_{w}^{2}}{\left(M_{2}-\mu\right)^{2}}+\frac{\left(M_{1} s_{\beta}-\left(M_{1}-2 \mu\right) c_{\beta}\right) s_{w}^{2}}{\left(M_{1}-\mu\right)^{2}}\right), \\
& x_{2}=\frac{\mu}{4 \sqrt{2}}\left(-\frac{\left(\left(M_{2}+2 \mu\right) c_{\beta}+M_{2} s_{\beta}\right) c_{w}^{2}}{\left(M_{2}+\mu\right)^{2}}-\frac{\left(\left(M_{1}+2 \mu\right) c_{\beta}+M_{1} s_{\beta}\right) s_{w}^{2}}{\left(M_{1}+\mu\right)^{2}}\right), \\
& x_{3}=\frac{\mu}{4 \sqrt{2}}\left(\frac{\left(\left(M_{2}-2 \mu\right) s_{\beta}-M_{2} c_{\beta}\right) c_{w}^{2}}{\left(M_{2}-\mu\right)^{2}}+\frac{\left(\left(M_{1}-2 \mu\right) s_{\beta}-M_{1} c_{\beta}\right) s_{w}^{2}}{\left(M_{1}-\mu\right)^{2}}\right), \\
& x_{4}=\frac{\mu}{4 \sqrt{2}}\left(\frac{\left(M_{2} c_{\beta}+\left(M_{2}+2 \mu\right) s_{\beta}\right) c_{w}^{2}}{\left(M_{2}+\mu\right)^{2}}+\frac{\left(M_{1} c_{\beta}+\left(M_{1}+2 \mu\right) s_{\beta}\right) s_{w}^{2}}{\left(M_{1}+\mu\right)^{2}}\right) .
\end{aligned}
$$

The numerical error of the matrix (A.12) in our parameter range of interest is smaller than $40 \%$ of the given NLO term. We do not discuss the slow convergence for this R-parity conserving sub-matrix further, since this is beyond the scope of our analysis.

Furthermore,

$$
\begin{aligned}
& U_{a i}^{\left(\chi^{0}, \nu\right)}=\zeta_{i}\left(\begin{array}{c}
s_{w} \frac{m_{Z}}{M_{1}} \\
-c_{w} \frac{m_{Z}}{M_{2}} \\
-\frac{m_{Z}^{2} c_{\beta}\left(M_{1} c_{w}^{2}+M_{2} s_{w}^{2}\right)}{M_{1} M_{2} \mu} \\
\frac{m_{Z}^{2} s_{\beta}\left(M_{1} c_{w}^{2}+M_{2} s_{w}^{2}\right)}{M_{1} M_{2} \mu}
\end{array}\right)\left(1+\mathcal{O}\left(s_{2 \beta} \frac{m_{Z}^{2}}{\mu^{2}}\right)\right), \\
& -s_{w} \frac{m_{Z}}{M_{1}} \\
& c_{w} \frac{m_{Z}}{M_{2}} \\
& U_{i a}^{\left(\nu, \chi^{0}\right)}=\zeta_{i}\left(\begin{array}{c}
\left(\begin{array}{c}
m_{Z}{ }^{2}\left(M_{1} c_{w}^{2}+M_{2} s_{w}^{2}-\mu\right)\left(c_{\beta}+s_{\beta}\right) \\
\sqrt{2}\left(M_{1}-\mu\right) \mu\left(\mu-M_{2}\right)
\end{array}\right. \\
\frac{m_{Z}\left(M_{1} c_{w}^{2}+M_{2} s_{w}^{2}+\mu\right)\left(c_{\beta}-s_{\beta}\right)}{\sqrt{2} \mu\left(M_{1}+\mu\right)\left(M_{2}+\mu\right)}
\end{array}\right)\left(1+\mathcal{O}\left(\frac{m_{Z}^{2}}{\mu^{2}}\right)\right), \\
& U_{i j}^{(\nu)}=\delta_{i j}+\mathcal{O}\left(\zeta^{2} \frac{m_{Z}^{2}}{\mu^{2}}\right) .
\end{aligned}
$$

The uncertainties in eq. (A.17) evaluate numerically to less than $5 \%$. For $U_{i a}^{\left(\nu, \chi^{0}\right)}$ they are less than $0.15,0.10,0.25,0.25$, for $a=1, \ldots, 4$, respectively. 
The unitary matrices $U^{(c)}$ and $\widetilde{U}^{(c)}$ which diagonalize the matrix $\mathcal{M}^{C}$, cf. eq. (A.2), can be denoted as

$$
\widetilde{U}^{(c)}=\left(\begin{array}{c|c}
\widetilde{U}_{\alpha \beta}^{\left(\chi^{+}\right)} & \widetilde{U}_{\alpha i}^{\left(\chi^{+}, e^{c}\right)} \\
\hline \widetilde{U}_{i \alpha}^{\left(e^{c}, \chi^{+}\right)} & \widetilde{U}_{i j}^{\left(e^{c}\right)}
\end{array}\right), \quad U^{(c)}=\left(\begin{array}{c|c}
U_{\alpha \beta}^{\left(\chi^{-}\right)} & U_{\alpha i}^{\left(\chi^{-}, e\right)} \\
\hline U_{i \alpha}^{\left(e, \chi^{-}\right)} & U_{i j}^{(e)}
\end{array}\right) .
$$

We find

$$
\begin{aligned}
& \widetilde{U}_{\alpha \beta}^{\left(\chi^{+}\right)}=\left(\begin{array}{ll}
1 & 0 \\
0 & 1
\end{array}\right)+\left(\begin{array}{cc}
-\frac{m_{Z}{ }^{2} c_{w}^{2}\left(\mu c_{\beta}+M_{2} s_{\beta}\right)^{2}}{2\left(M_{2}{ }^{2}-\mu^{2}\right)^{2}} & -\frac{m_{Z} c_{w}\left(\mu c_{\beta}+M_{2} s_{\beta}\right)}{M_{2}{ }^{2}-\mu^{2}} \\
\frac{m_{Z} c_{w}\left(\mu c_{\beta}+M_{2} s_{\beta}\right)}{M_{2}{ }^{2}-\mu^{2}} & -\frac{m_{Z}{ }^{2} c_{w}^{2}\left(\mu c_{\beta}+M_{2} s_{\beta}\right)^{2}}{2\left(M_{2}{ }^{2}-\mu^{2}\right)^{2}}
\end{array}\right)\left(1+\mathcal{O}\left(\frac{m_{Z}^{2}}{\mu^{2}}\right)\right), \\
& \widetilde{U}_{i j}^{\left(e^{c}\right)}=\delta_{i j}+\mathcal{O}\left(\zeta^{2}\right) .
\end{aligned}
$$

Numerically, the relative correction to the NLO contribution to $\widetilde{U}_{\alpha \beta}^{\left(\chi^{+}\right)}$is less than $25 \%$. The off-diagonal elements of the matrix $\widetilde{U}^{(c)}$ to leading order in $h_{i i}^{e}$ are

$$
\begin{aligned}
\widetilde{U}_{\alpha i}^{\left(\chi^{+}, e^{c}\right)}= & -\zeta_{i} h_{i i}^{e}\left(\begin{array}{l}
0 \\
1
\end{array}\right) \\
& +\zeta_{i} h_{i i}^{e}\left(\begin{array}{c}
\frac{m_{Z} c_{w}\left(M_{2} s_{\beta}-v c_{\beta}\right)}{M_{2}^{2}} \\
\frac{m_{Z}^{2} c_{\beta} c_{w}^{2}\left(v \mu c_{\beta}+M_{2}(v-\mu) s_{\beta}\right)}{M_{2}^{2} \mu^{2}}
\end{array}\right)\left(1+\mathcal{O}\left(\begin{array}{c}
\frac{s_{2 \beta} m_{Z}^{2}}{\mu^{2}} \\
\frac{m_{Z}^{2}}{\mu^{2}}
\end{array}\right)\right), \\
\widetilde{U}_{i \alpha}^{\left(e^{c}, \chi^{+}\right)}= & \zeta_{i} h_{i i}^{e}\left(\begin{array}{l}
0 \\
1
\end{array}\right) \\
& +\zeta_{i} h_{i i}^{e}\left(\begin{array}{c}
\frac{m_{Z} c_{w}\left(M_{2} s_{\beta} \mu^{2}+\left(M_{2}{ }^{2}(v+\mu)-v \mu^{2}\right) c_{\beta}\right)}{M_{2}{ }^{2}\left(M_{2}^{2}-\mu^{2}\right)} \\
-\frac{m_{Z} \mu^{2} c_{w}^{2} y}{2\left(\mu^{2}-M_{2}{ }^{2}\right)^{2}}
\end{array}\right)\left(1+\mathcal{O}\left(\frac{m_{Z}^{2}}{\mu^{2}}\right)\right),
\end{aligned}
$$

where

$$
\begin{aligned}
y= & \frac{1}{\mu^{4}}\left(v s_{2 \beta} M_{2}^{3}+\mu\left(2 v M_{2}^{2}+\mu^{2}(\mu-2 v)\right) c_{\beta}^{2}+\mu s_{\beta}\left(\mu\left(2 \mu^{2}-M_{2}^{2}\right) s_{\beta}\right.\right. \\
& \left.\left.-2 M_{2}\left(M_{2}^{2}+(v-2 \mu) \mu\right) c_{\beta}\right)\right) .
\end{aligned}
$$

The numerical relative correction to the NLO term in $\widetilde{U}_{i \alpha}^{\left(e^{c}, \chi^{+}\right)}$is smaller than $0.10,0.15$ for $\alpha=1,2$, respectively. For $\widetilde{U}_{1 i}^{\left(\chi^{+}, e^{c}\right)}$ it is smaller ${ }^{8}$ than $1 \%$, and smaller than $10 \%$ for $\widetilde{U}_{2 i}^{\left(\chi^{+}, e^{c}\right)}$.

The block diagonal elements of the matrix $U^{(c)}$ are

$$
\begin{aligned}
U_{\alpha \beta}^{\left(\chi^{-}\right)} & =\left(\begin{array}{ll}
1 & 0 \\
0 & 1
\end{array}\right)+\left(\begin{array}{ll}
-\frac{m_{Z}^{2} c_{w}^{2}\left(M_{2} c_{\beta}+\mu s_{\beta}\right)^{2}}{2\left(M_{2}^{2}-\mu^{2}\right)^{2}} & -\frac{m_{Z} c_{w}\left(M_{2} c_{\beta}+\mu s_{\beta}\right)}{M_{2}^{2}-\mu^{2}} \\
\frac{m_{Z} c_{w}\left(M_{2} c_{\beta}+\mu s_{\beta}\right)}{M_{2}^{2}-\mu^{2}} & -\frac{m_{Z} c_{w}^{2}\left(M_{2} c_{\beta}+\mu s_{\beta}\right)^{2}}{2\left(M_{2}^{2}-\mu^{2}\right)^{2}}
\end{array}\right)\left(1+\mathcal{O}\left(\frac{m_{Z}^{2}}{\mu^{2}}\right)\right), \\
U_{i j}^{(e)} & =\delta_{i j}+\mathcal{O}\left(\zeta^{2}\right) .
\end{aligned}
$$

\footnotetext{
${ }^{8}$ The numerical calculation of the error reaches our numerical precision. The given value is calculated from the comparison with the analytical NNLO expression.
} 
Numerically, the relative correction to the NLO contribution to $U_{\alpha \beta}^{\left(\chi^{-}\right)}$is smaller than $20 \%$. The off-diagonal elements of $U^{(c)}$ are

$$
\begin{aligned}
& U_{\alpha i}^{\left(\chi^{-}, e\right)}=\zeta_{i}\left(\begin{array}{c}
-\frac{m_{Z} c_{w}}{M_{2}} \\
\frac{m_{Z}{ }^{2} s_{\beta}}{M_{2} \mu}
\end{array}\right)\left(1+\mathcal{O}\left(\frac{s_{2 \beta} m_{Z}^{2}}{\mu^{2}}\right)\right), \\
& U_{i \alpha}^{\left(e, \chi^{-}\right)}=\zeta_{i}\left(\begin{array}{c}
\frac{m_{Z} c_{w}}{M_{2}} \\
\frac{m_{Z}{ }^{2} c_{w}^{2}\left(\mu c_{\beta}+M_{2} s_{\beta}\right)}{\mu^{3}-M_{2}{ }^{2} \mu}
\end{array}\right)\left(1+\mathcal{O}\left(\frac{s_{2 \beta} m_{Z}^{2}}{\mu^{2}}\right)\right) .
\end{aligned}
$$

Here we ignored corrections that are proportional to the Yukawa couplings $h_{i i}^{e}$ or higher powers thereof. The numerical value of the higher order correction relative to the NLO term is smaller than $1 \%$ for $U_{\alpha i}^{\left(\chi^{-}, e\right)}$, smaller than $5 \%$ for $U_{i 1}^{\left(e, \chi^{-}\right)}$, and smaller than $15 \%$ for $U_{i 2}^{\left(e, \chi^{-}\right)}$.

\section{A.2 The currents in mass eigenstate basis}

The neutral and charged currents were given in eqs. (4.11) and (4.12),

$$
\begin{aligned}
J_{Z \mu}= & \bar{\chi}_{a}^{0} \gamma_{\mu} V_{a b}^{\left(\chi^{0}\right)} \chi_{b}^{0}+\bar{\chi}_{\alpha}^{-} \gamma_{\mu} V_{\alpha \beta}^{\left(\chi^{-}\right)} \chi_{\beta}^{-}+\bar{\chi}_{\alpha}^{+} \gamma_{\mu} V_{\alpha \beta}^{\left(\chi^{+}\right)} \chi_{\beta}^{+}+\bar{\nu}_{i} \gamma_{\mu} V_{i j}^{(\nu)} \nu_{i}+\bar{e}_{i} \gamma_{\mu} V_{i j}^{(e)} e_{i} \\
& +\left(\bar{\chi}_{a}^{0} \gamma_{\mu} V_{a i}^{(\chi, \nu)} \nu_{i}+\bar{\chi}_{\alpha}^{-} \gamma_{\mu} V_{\alpha i}^{\left(\chi^{-}, e\right)} e_{i}+\bar{\chi}_{\alpha}^{+} \gamma_{\mu} V_{\alpha i}^{\left(\chi^{+}, e^{c}\right)} e_{i}^{c}+\text { h.c. }\right) \\
J_{\mu}^{-}= & \bar{\chi}_{a}^{0} \gamma_{\mu} V_{a \alpha}^{(\chi)} \chi_{\alpha}^{-}+\bar{\chi}_{a}^{0} \gamma_{\mu} V_{a i}^{(\chi, e)} e_{i}+\bar{\nu}_{i} \gamma_{\mu} V_{i \alpha}^{(\nu, \chi)} \chi_{\alpha}^{-}+\bar{\nu}_{i} \gamma_{\mu} V_{i j}^{(\nu, e)} e_{j} .
\end{aligned}
$$

The CKM-like matrices $V_{a b}^{\left(\chi^{0}\right)}, V_{\alpha \beta}^{\left(\chi^{-}\right)}, V_{\alpha \beta}^{\left(\chi^{+}\right)}, V_{i j}^{(\nu)}, V_{i j}^{(e)}, V_{a i}^{(\chi, \nu)}, V_{\alpha i}^{\left(\chi^{-}, e\right)}, V_{\alpha i}^{\left(\chi^{+}, e^{c}\right)}, V_{a \alpha}^{(\chi)}$, $V_{a i}^{(\chi, e)}, V_{i \alpha}^{(\nu, \chi)}, V_{i j}^{(\nu, e)}$ follow from the currents in gauge eigenbasis, eqs. (4.3) and (4.4), and the explicit matrices $U^{(n)}, U^{(c)}$ and $\widetilde{U}^{(c)}$. Here we focus on the matrices $V_{a i}^{(\chi, \nu)}$ and $V_{a i}^{(\chi, e)}$ since they determine the interactions of interest for this work. We find

$$
V_{a i}^{(\chi, \nu)}=\zeta_{i}\left(\begin{array}{c}
-\frac{s_{w} m_{Z}}{2 M_{1}} \\
\frac{c_{w} m_{Z}}{2 M_{2}} \\
\frac{m_{Z}^{2} \mu v_{1}}{2 \sqrt{2} M_{1}\left(M_{1}-\mu\right)\left(M_{2}-\mu\right)} \\
\frac{m_{Z}^{2} \mu v_{2}}{2 \sqrt{2} M_{1}\left(M_{1}+\mu\right)\left(M_{2}+\mu\right)}
\end{array}\right)\left(1+\mathcal{O}\left(\begin{array}{c}
\frac{s_{2 \beta} m_{Z}^{2}}{\mu^{2}} \\
\frac{m_{Z}^{2}}{\mu^{2}} \\
\frac{m_{Z}^{2}}{\mu^{2}} \\
\frac{s_{2 \beta} m_{Z}^{2}}{\mu^{2}}
\end{array}\right)\right),
$$

with abbreviations

$$
\begin{aligned}
v_{1}= & \frac{1}{M_{2} \mu^{2}}\left(M_{1}\left(M_{1}-\mu\right)\left(\left(\mu-2 M_{2}\right) c_{\beta}-\mu s_{\beta}\right) c_{w}^{2}\right. \\
& \left.+M_{2}\left(M_{2}-\mu\right)\left(\left(\mu-2 M_{1}\right) c_{\beta}-\mu s_{\beta}\right) s_{w}^{2}\right), \\
v_{2}= & \frac{1}{M_{2} \mu^{2}}\left(M_{1}\left(M_{1}+\mu\right)\left(\left(2 M_{2}+\mu\right) c_{\beta}+\mu s_{\beta}\right) c_{w}^{2}\right. \\
& \left.+M_{2}\left(M_{2}+\mu\right)\left(\left(2 M_{1}+\mu\right) c_{\beta}+\mu s_{\beta}\right) s_{w}^{2}\right) .
\end{aligned}
$$

Numerically, the relative errors are smaller than $0.10,0.20,0.15,0.05$ for $a=1, \ldots, 4$. 
Finally,

$$
V_{a i}^{(\chi, e)}=\zeta_{i}\left(\begin{array}{c}
-\frac{s_{w} m_{Z}}{M_{1}} \\
-\frac{(\sqrt{2}-1) c_{w} m_{Z}}{M_{2}} \\
-\frac{m_{Z}^{2} \mu \tilde{v}_{1}}{M_{2}\left(M_{1}-\mu\right)\left(M_{2}-\mu\right)} \\
\frac{m_{Z}^{2} \mu \tilde{v}_{2}}{M_{2}\left(M_{1}+\mu\right)\left(M_{2}+\mu\right)}
\end{array}\right)\left(1+\mathcal{O}\left(\begin{array}{c}
\frac{s_{2 \beta} m_{Z}^{2}}{\mu^{2}} \\
\frac{m_{Z}^{2}}{\mu^{2}} \\
\frac{m_{Z}^{2}}{\mu^{2}} \\
\frac{m_{Z}^{2}}{\mu^{2}}
\end{array}\right)\right),
$$

with abbreviations

$$
\begin{aligned}
\tilde{v}_{1}= & \frac{1}{2 \mu^{2}}\left(c_{\beta}\left(\left(M_{1}-\mu\right)\left(\sqrt{2} M_{2}-2 \mu\right) c_{w}^{2}+\sqrt{2} M_{2}\left(M_{2}-\mu\right) s_{w}^{2}\right)\right. \\
& \left.+s_{\beta}\left((\sqrt{2}-2)\left(M_{1}-\mu\right) \mu c_{w}^{2}+\sqrt{2} M_{2}\left(M_{2}-\mu\right) s_{w}^{2}\right)\right) \\
\tilde{v}_{2}= & \frac{1}{2 \mu^{2}}\left(c_{\beta}\left(\left(M_{1}+\mu\right)\left(\sqrt{2} M_{2}+2 \mu\right) c_{w}^{2}+\sqrt{2} M_{2}\left(M_{2}+\mu\right) s_{w}^{2}\right)\right. \\
& \left.+s_{\beta}\left((\sqrt{2}-2) \mu\left(M_{1}+\mu\right) c_{w}^{2}-\sqrt{2} M_{2}\left(M_{2}+\mu\right) s_{w}^{2}\right)\right) .
\end{aligned}
$$

Here we again neglected corrections that involve the Yukawa couplings $h_{i i}^{e}$. The numerical corrections to the NLO contributions to $V_{a i}^{(\chi, e)}$ are smaller than $0.05,0.15,0.20$ for $a=$ $1,2,3$, respectively. For $a=4$ we reach the limit of our numerical precision.

Open Access. This article is distributed under the terms of the Creative Commons Attribution Noncommercial License which permits any noncommercial use, distribution, and reproduction in any medium, provided the original author(s) and source are credited.

\section{References}

[1] D.Z. Freedman, P. van Nieuwenhuizen and S. Ferrara, Progress Toward a Theory of Supergravity, Phys. Rev. D 13 (1976) 3214 [SPIRES].

[2] S. Deser and B. Zumino, Consistent Supergravity, Phys. Lett. B 62 (1976) 335 [SPIRES].

[3] H. Pagels and J.R. Primack, Supersymmetry, Cosmology and New TeV Physics, Phys. Rev. Lett. 48 (1982) 223 [SPIRES].

[4] S. Weinberg, Cosmological Constraints on the Scale of Supersymmetry Breaking, Phys. Rev. Lett. 48 (1982) 1303 [SPIRES].

[5] J.R. Ellis, D.V. Nanopoulos and S. Sarkar, The Cosmology of Decaying Gravitinos, Nucl. Phys. B 259 (1985) 175 [SPIRES].

[6] M. Kawasaki, K. Kohri and T. Moroi, Hadronic decay of late-decaying particles and big-bang nucleosynthesis, Phys. Lett. B 625 (2005) 7 [astro-ph/0402490] [SPIRES].

[7] M. Kawasaki, K. Kohri and T. Moroi, Big-bang nucleosynthesis and hadronic decay of long-lived massive particles, Phys. Rev. D 71 (2005) 083502 [astro-ph/0408426] [SPIRES].

[8] K. Jedamzik, Big bang nucleosynthesis constraints on hadronically and electromagnetically decaying relic neutral particles, Phys. Rev. D 74 (2006) 103509 [hep-ph/0604251] [SPIRES].

[9] M. Fukugita and T. Yanagida, Baryogenesis Without Grand Unification, Phys. Lett. B 174 (1986) 45 [SPIRES]. 
[10] M. Bolz, W. Buchmüller and M. Plümacher, Baryon asymmetry and dark matter, Phys. Lett. B 443 (1998) 209 [hep-ph/9809381] [SPIRES].

[11] J.L. Feng, Supersymmetry and cosmology, Annals Phys. 315 (2005) 2 [SPIRES].

[12] W. Buchmüller, L. Covi, K. Hamaguchi, A. Ibarra and T. Yanagida, Gravitino dark matter in R-parity breaking vacua, JHEP 03 (2007) 037 [hep-ph/0702184] [SPIRES].

[13] F. Takayama and M. Yamaguchi, Gravitino dark matter without R-parity, Phys. Lett. B 485 (2000) 388 [hep-ph/0005214] [SPIRES].

[14] S. Lola, P. Osland and A.R. Raklev, Radiative gravitino decays from R-parity violation, Phys. Lett. B 656 (2007) 83 [arXiv:0707.2510] [SPIRES].

[15] G. Bertone, W. Buchmüller, L. Covi and A. Ibarra, Gamma-Rays from Decaying Dark Matter, JCAP 11 (2007) 003 [arXiv: 0709. 2299] [SPIRES].

[16] A. Ibarra and D. Tran, Gamma Ray Spectrum from Gravitino Dark Matter Decay, Phys. Rev. Lett. 100 (2008) 061301 [arXiv:0709.4593] [SPIRES].

[17] K. Ishiwata, S. Matsumoto and T. Moroi, High Energy Cosmic Rays from the Decay of Gravitino Dark Matter, Phys. Rev. D 78 (2008) 063505 [arXiv:0805.1133] [SPIRES].

[18] W. Buchmüller, A. Ibarra, T. Shindou, F. Takayama and D. Tran, Probing Gravitino Dark Matter, JCAP 09 (2009) 021 [arXiv: 0906.1187] [SPIRES].

[19] N.E. Bomark, S. Lola, P. Osland and A.R. Raklev, Photon, Neutrino and Charged Particle Spectra from R-violating Gravitino Decays, Phys. Lett. B 686 (2010) 152 [arXiv:0911.3376] [SPIRES].

[20] K.-Y. Choi, D. Restrepo, C.E. Yaguna and O. Zapata, Indirect detection of gravitino dark matter including its three-body decays, arXiv:1007.1728 [SPIRES].

[21] A.A. Abdo et al., Fermi LAT Search for Photon Lines from 30 to $200 \mathrm{GeV}$ and Dark Matter Implications, Phys. Rev. Lett. 104 (2010) 091302 [arXiv:1001.4836] [SPIRES].

[22] The Fermi-LAT collaboration, A.A. Abdo et al., The Spectrum of the Isotropic Diffuse Gamma-Ray Emission Derived From First-Year Fermi Large Area Telescope Data, Phys. Rev. Lett. 104 (2010) 101101 [arXiv:1002.3603] [SPIRES].

[23] W. Buchmüller, M. Endo and T. Shindou, Superparticle Mass Window from Leptogenesis and Decaying Gravitino Dark Matter, JHEP 11 (2008) 079 [arXiv:0809.4667] [SPIRES].

[24] L.J. Hall and M. Suzuki, Explicit R-Parity Breaking in Supersymmetric Models, Nucl. Phys. B 231 (1984) 419 [SPIRES].

[25] B.C. Allanach, A. Dedes and H.K. Dreiner, The $R$ parity violating minimal supergravity model, Phys. Rev. D 69 (2004) 115002 [Erratum ibid. D 72 (2005) 079902] [hep-ph/0309196] [SPIRES].

[26] R. Barbier et al., R-parity violating supersymmetry, Phys. Rept. 420 (2005) 1 [hep-ph/0406039] [SPIRES].

[27] F. de Campos et al., Probing bilinear R-parity violating supergravity at the LHC, JHEP 05 (2008) 048 [arXiv:0712.2156] [SPIRES].

[28] B.C. Allanach and M.A. Bernhardt, Including R-parity violation in the numerical computation of the spectrum of the minimal supersymmetric standard model: SOFTSUSY3.0, Comput. Phys. Commun. 181 (2010) 232 [arXiv:0903.1805] [SPIRES]. 
[29] B.A. Campbell, S. Davidson, J.R. Ellis and K.A. Olive, Cosmological baryon asymmetry constraints on extensions of the standard model, Phys. Lett. B 256 (1991) 484 [SPIRES].

[30] W. Fischler, G.F. Giudice, R.G. Leigh and S. Paban, Constraints on the baryogenesis scale from neutrino masses, Phys. Lett. B 258 (1991) 45 [SPIRES].

[31] H.K. Dreiner and G.G. Ross, Sphaleron erasure of primordial baryogenesis, Nucl. Phys. B 410 (1993) 188 [hep-ph/9207221] [SPIRES].

[32] M. Endo, K. Hamaguchi and S. Iwamoto, Lepton Flavor Violation and Cosmological Constraints on R-parity Violation, JCAP 02 (2010) 032 [arXiv:0912.0585] [SPIRES].

[33] G.F. Giudice and A. Masiero, A Natural Solution to the mu Problem in Supergravity Theories, Phys. Lett. B 206 (1988) 480 [SPIRES].

[34] W. Buchmüller and T. Yanagida, Quark lepton mass hierarchies and the baryon asymmetry, Phys. Lett. B 445 (1999) 399 [hep-ph/9810308] [SPIRES].

[35] W. Buchmüller, D. Delepine and L.T. Handoko, Neutrino mixing and flavor changing processes, Nucl. Phys. B 576 (2000) 445 [hep-ph/9912317] [SPIRES].

[36] M. Grefe, Neutrino signals from gravitino dark matter with broken R-parity, DESY-THESIS-2008-043 [SPIRES].

[37] B. Mukhopadhyaya, S. Roy and F. Vissani, Correlation between neutrino oscillations and collider signals of supersymmetry in an R-parity violating model, Phys. Lett. B 443 (1998) 191 [hep-ph/9808265] [SPIRES].

[38] E.J. Chun and J.S. Lee, Implication of Super-Kamiokande data on R-parity violation, Phys. Rev. D 60 (1999) 075006 [hep-ph/9811201] [SPIRES].

[39] K. Ishiwata, T. Ito and T. Moroi, Long-Lived Unstable Superparticles at the LHC, Phys. Lett. B 669 (2008) 28 [arXiv:0807.0975] [SPIRES].

[40] S. Asai, K. Hamaguchi and S. Shirai, Stop and Decay of Long-lived Charged Massive Particles at the LHC detectors, Phys. Rev. Lett. 103 (2009) 141803 [arXiv:0902.3754] [SPIRES].

[41] W. Buchmüller, K. Hamaguchi, M. Ratz and T. Yanagida, Supergravity at colliders, Phys. Lett. B 588 (2004) 90 [hep-ph/0402179] [SPIRES]. 